\title{
Regulation of Neuronal M-Channel Gating in an Isoform-Specific Manner: Functional Interplay between Calmodulin and Syntaxin 1A
}

\author{
Adi Etzioni, ${ }^{1 \star}$ Sivan Siloni, ${ }^{1 \star}$ Dodo Chikvashvilli, ${ }^{1}$ Roi Strulovich, ${ }^{2}$ Dana Sachyani, ${ }^{2}$ Noa Regev, ${ }^{1}$ Dafna Greitzer-Antes, ${ }^{1}$ \\ Joel A. Hirsch, ${ }^{2}$ and Ilana Lotan ${ }^{1}$ \\ ${ }^{1}$ Department of Physiology and Pharmacology, Sackler School of Medicine, and ${ }^{2}$ Department of Biochemistry, Faculty of Life Sciences, Tel Aviv University, \\ Ramat Aviv 69978, Israel
}

Whereas neuronal M-type $\mathrm{K}^{+}$channels composed of KCNQ2 and KCNQ3 subunits regulate firing properties of neurons, presynaptic KCNQ2 subunits were demonstrated to regulate neurotransmitter release by directly influencing presynaptic function. Two interaction partners of M-channels, syntaxin $1 \mathrm{~A}$ and calmodulin, are known to act presynaptically, syntaxin serving as a major protein component of the membrane fusion machinery and calmodulin serving as regulator of several processes related to neurotransmitter release. Notably, both partners specifically modulate KCNQ2 but not KCNQ3 subunits, suggesting selective presynaptic targeting to directly regulate exocytosis without interference in neuronal firing properties. Here, having first demonstrated in Xenopus oocytes, using analysis of single-channel biophysics, that both modulators downregulate the open probability of KCNQ2 but not KCNQ3 homomers, we sought to resolve the channel structural determinants that confer the isoform-specific gating downregulation and to get insights into the molecular events underlying this mechanism. We show, using optical, biochemical, electrophysiological, and molecular biology analyses, the existence of constitutive interactions between the N and C termini in homomeric KCNQ2 and KCNQ3 channels in living cells. Furthermore, rearrangement in the relative orientation of the KCNQ2 termini that accompanies reduction in single-channel open probability is induced by both regulators, strongly suggesting that closer $\mathrm{N}-\mathrm{C}$ termini proximity underlies gating downregulation. Different structural determinants, identified at the $\mathrm{N}$ and $\mathrm{C}$ termini of $\mathrm{KCNQ}$, prevent the effects by syntaxin $1 \mathrm{~A}$ and calmodulin, respectively. Moreover, we show that the syntaxin $1 \mathrm{~A}$ and calmodulin effects can be additive or blocked at different concentration ranges of calmodulin, bearing physiological significance with regard to presynaptic exocytosis.

\section{Introduction}

M-channels comprise members of the Kv7 family of voltagedependent potassium channels expressed exclusively in the nervous system, mainly KCNQ2 (Q2) and KCNQ3 (Q3), with possible additional contribution from KCNQ4 and KCNQ5 at specific neuronal sites (Brown and Adams, 1980; Wang et al., 1998; Jentsch, 2000; Robbins, 2001). Whereas heteromeric Q2/Q3 channels are expressed in cell bodies and dendrites of many hippocampal and cortical neurons (Marrion, 1997; Cooper and Jan, 2003) and also in the initial segment of several neurons

\footnotetext{
Received May 27, 2011; revised Aug. 2, 2011; accepted Aug. 9, 2011

Author contributions: A.E., S.S., D.C., and I.L. designed research; A.E., S.S., D.C., R.S., D.S., and D.G.-A. performed research; A.E., S.S., R.S., and D.S. contributed unpublished reagents/analytic tools; A.E., S.S., and D.G.-A. analyzed data; A.E., S.S., N.R., J.A.H., and I.L. wrote the paper.

This work was supported by grants from the Israel Academy of Sciences (I.L.) and the State of Israel Ministry of Health (I.L.). We thank Thomas Jentsch for the Q2-HA and Q3-HA cDNAs and Alvaro Villarroel for the Q3*, $2_{N} / \mathrm{Q3}_{\mathrm{C}^{*}}^{*}$ and $Q 2_{N} / Q 3_{C}^{*}$ CDNAs. Kv2.1 was kindly provided by Prof. R. Joho (University of Texas Southwestern Medical Center, Dallas, TX).

The authors declare no competing financial interests.

*A.E. and S.S. contributed equally to this work.

Correspondence should be addressed to llana Lotan, Department of Physiology and Pharmacology, Sackler School of Medicine, Tel Aviv University, Ramat Aviv 69978, Israel. E-mail: ilotan@post.tau.ac.il.

DOI:10.1523/JNEUROSCI.2666-11.2011

Copyright $\odot 2011$ the authors $\quad 0270-6474 / 11 / 3114158-14 \$ 15.00 / 0$
}

(Devaux et al., 2004; Chung et al., 2006), where they control neuronal excitability by limiting repetitive firing (Hille, 1992; Dolly and Parcej, 1996; Miller, 2000; Millar et al., 2007; Regev et al., 2009), exclusive expression of Q2 subunits can be found in nerve terminals, where homomeric Q2 channels might directly regulate neurotransmitter release (Cooper et al., 2001; Devaux et al., 2004; Martire et al., 2004; Peretz et al., 2007).

Activation of G-protein-coupled receptors of the Gq/11 family strongly suppresses M-channels (Delmas and Brown, 2005), via depletion of phosphatidylinositol 4,5-bisphosphate $\left(\mathrm{PIP}_{2}\right)$ upon muscarinic receptor stimulation (Suh and Hille, 2002; Ford et al., 2003; Zhang et al., 2003; Suh et al., 2004; Li et al., 2005; Winks et al., 2005) or via $\mathrm{Ca}^{2+}$-calmodulin (CaM) action on the channels upon bradykinin $\mathrm{B}_{2}$ and purinergic $\mathrm{P}_{2} \mathrm{Y}$ receptor activation (Cruzblanca et al., 1998; Bofill-Cardona et al., 2000; Gamper and Shapiro, 2003; Zaika et al., 2007). Recently, syntaxin 1A (Syx), a plasma membrane protein component of the exocytotic SNARE complex, shown to interact physically and functionally with different voltage-gated $\mathrm{K}^{+}$ channels (Fili et al., 2001; Leung et al., 2003, 2007; Michaelevski et al., 2003, 2007; Tsuk et al., 2005; Yamakawa et al., 2007; Regev et al., 2009), was suggested to be another blocker of M-channels (Regev et al., 2009). 
Notably, CaM, $\mathrm{PIP}_{2}$, and Syx were shown to selectively affect Q2 but not Q3 subunits and to bind at a membrane-proximal C-terminal cytoplasmic region comprising of helices A and B (Vizi et al., 2000; Wen and Levitan, 2002; Yus-Najera et al., 2002; Gamper and Shapiro, 2003; Li et al., 2005; Hernandez et al., 2008; Regev et al., 2009). Mutations in this region can lead to dominant inherited human epilepsy (benign neonatal familial convulsions; Biervert et al., 1998; Singh et al., 1998). Although all three modulators have been suggested to affect Q2 channel gating (Gamper et al., 2005; Li et al., 2005; Regev et al., 2009), this was directly demonstrated only with $\mathrm{PIP}_{2}$ (Li et al., 2005). Moreover, the molecular mechanism that controls the gating is still unknown.

Here, we characterize the effects of Syx and CaM on Q2, Q3, and chimeric channels, using electrophysiological analyses at the levels of whole cell and single channel, combined with fluorescence resonance energy transfer (FRET) and biochemical analyses. We put forward a mechanism for M-channel gating regulation in an isoform-specific manner that involves rearrangement in the relative orientation of cytoplasmic domains. Notably, although this same mechanism is used by both proteins for Q2 regulation, it is prevented in Q3 by channel determinants different for each of the modulators. Finally, we show complex functional interplay between the two modulators.

\section{Materials and Methods}

\section{Constructs and antibodies}

Human Q2 (Y15065), Q3 (NM004519), Q2-HA, and Q3-HA (tagged with HA epitope in the extracellular loop that contacts transmembrane domains S1 and S2) cDNAs were kindly provided by Thomas Jentsch (Zentrum für Molekulare Neuropathobiologie, Hamburg, Germany). $\mathrm{Q}^{*}, \mathrm{Q} 2_{\mathrm{N}} / \mathrm{Q} 3_{\mathrm{C}}$, and $\mathrm{Q} 2_{\mathrm{N}} / \mathrm{Q} 3_{\mathrm{C}}$ were kindly provided by Alvaro Villarroel (Unidad de Biofísica, Consejo Superior de Investigaciones CientíficasUniversidad del País Vasco (UPV)/Euskal Herriko Unibersitatea, UPV, Leioa, Spain). All chimeras were constructed by exchanging equivalent regions of Q2 and Q3, using direct cloning of PCR (Weiner, 1993). Deletions mutants were constructed using QuikChange Site-Directed Mutagenesis kit (Stratagene). Double- and single-labeled Q2 or Q3* were constructed by subcloning of native channels into pGEMHJ containing enhanced CFP (eCFP) and enhanced YFP (eYFP) at N terminus (NT) and C terminus (CT) positions, respectively, via Ser-Arg linkers (encoded by XbaI restriction sites). eYFP had the Q69M mutation reducing its environmental sensitivity (Griesbeck et al., 2001). Both eCFP and eYFP had the A206K mutation, reducing their affinity to each other (Shaner et al., 2005). Mutant Kv2.1 channels Kv2.1 $\Delta$ C1a (amino acids 421-523 deleted) and Kv2.1 $\Delta 331$ (amino acids 523-854 deleted) were created using two-step overlapping PCR with wild-type rat Kv2.1 cDNA as the template and subcloned into the EcoRI/XbaI sites of the pGEMHJ vector was created using two-step overlapping PCR with wild-type rat Kv2.1 cDNA as the template and subcloned into the EcoRI/XbaI sites of the pGEMHJ vector. $\mathrm{Kv} 2.1 \Delta \mathrm{C} 1 \mathrm{a}-\mathrm{Y}$ was created by fusing eYFP to the CT of the channel subunits, using two-step overlapping PCR via Pro-Gly linkers (encoded by AvaI restriction sites). Kv2.1 $331-\mathrm{Y}$ was created by fusing eYFP to the CT of the channel subunits, using three-step overlapping PCR (Wurch et al., 1998). C-Syx was created by fusing eCFP to the NT of Syx, via Leu-Glu linkers (encoded by XhoI restriction sites). For bacterial expression of Q2 CT, the human KCNQ2 sequence was used as a template for PCR with BamHI and NotI restriction sites added. PCR product (residues 316633) was ligated into a doubly digested (BamHI and NotI) modified pETDuet vector (Novagen), in which CaM was inserted into multiple cloning site II, as described previously (Weiner, 1993). All constructs were verified by DNA sequencing. Antibodies used included the following: anti-HA (Santa Cruz Biotechnology), monoclonal anti-calmodulin (Millipore), and anti-KCNQ2 and anti-KCNQ3 (Alomone Labs).

\section{Electrophysiology in oocytes}

Female Xenopus laevis oocytes were prepared (Dascal, 1993) and proteins were expressed as described previously (Levin et al., 1995). The following
mRNA concentrations were injected (in ng/oocyte): 7.5 Q2, 0.75 Q3, 0.5

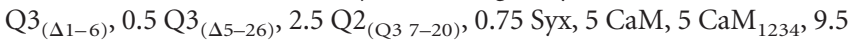
C-Q2-Y, 0.95 C-Q3*-Y, 0.95 C-Q3 ${ }_{(\Delta 1-6)}-\mathrm{Y}, 3.2$ C-Q2 $\left.{ }_{(\mathrm{Q} 3} 7-20\right)^{-} \mathrm{Y}$, unless otherwise stated in the figure legends (e.g., for high expression levels of channels in the CaM experiments, mRNA concentrations were multiplied by $\sim 2.5)$. Chimera $\mathrm{Q}_{(\mathrm{Q} 2}^{*}$ helixA) was coexpressed with $\mathrm{Q}^{*}$ to enhance expression.

Two-electrode voltage-clamp analysis of macro currents was performed as described previously (Levin et al., 1995; Regev et al., 2009). Antisense oligonucleotide for CaM (A.S. CaM) was injected (50 ng/ oocyte) $1 \mathrm{~d}$ before the channel mRNA injection and every $48 \mathrm{~h}$ until the experiment was performed. The degenerate phosphorothioate antisense oligodeoxynucleotide (including $5^{\prime}$ phosphorothioates at the first two positions and $3^{\prime}$ phosphorothioates at the last three positions, to enhance nuclease resistance) was targeted against the following nucleotide sequence: 5'-GTA CCA TCA CCA CAA AG-3' of the Xenopus laevis CaM gene (Chien and Dawid, 1984).

Data analysis. Current-voltage relationships were obtained by depolarizing steps (of $1.5 \mathrm{~s}$ ) from a holding potential of -90 to $+50 \mathrm{mV}$ in 10 $\mathrm{mV}$ increments (with $5 \mathrm{~s}$ intervals between episodes). Net current is obtained by subtracting the scaled leak current elicited by a voltage step from -90 to $-100 \mathrm{mV}$. Oocytes with a leak current of $>4 \mathrm{nA} / 1 \mathrm{mV}$ are discarded. The channel conductance was calculated as follows:

$$
g_{i}=\frac{I_{i}}{\left(V-E_{i}\right)}
$$

where $g_{i}$ is the conductance of the channel, $V$ is the membrane potential in each episode set with the voltage-clamp technique, and $E_{i}$ is a reversal potential for the specific ion $(-98 \mathrm{mV})$. The half-activation voltage $\left(V a_{1 / 2}\right)$ and slope factor $(K a)$ were derived by fitting the ratio $\mathrm{g} / \mathrm{g}_{\max }$ versus voltage to the Boltzmann equation for each tested oocytes as follows:

$$
\frac{g}{g_{\max }}=\frac{1}{1+\exp \left(-\left(V-V a_{1 / 2}\right) / K a\right)} .
$$

Single-channel patch clamp. Cell-attached patch-clamp analysis of single-channel currents was performed as described previously (SingerLahat et al., 1999; Michaelevski et al., 2007). Oocytes of which the vitelline membrane was removed were placed in a bath solution containing the following: $146 \mathrm{~mm} \mathrm{KCl}, 2 \mathrm{~mm} \mathrm{NaCl}, 1 \mathrm{mM} \mathrm{CaCl}_{2}, 1 \mathrm{~mm} \mathrm{MgCl}_{2}, 0.1 \mathrm{~mm}$ EGTA, 10 mM HEPES, $\mathrm{pH}$ 8. Patch pipettes were pulled from glass capillary tubing (Warner Instruments) with 2-5 M $\Omega$ tip resistance. Patch pipettes were filled with solution containing $150 \mathrm{~mm} \mathrm{NaCl}, 2 \mathrm{~mm} \mathrm{KCl}, 1$ $\mathrm{mm} \mathrm{CaCl}_{2}, 1 \mathrm{~mm} \mathrm{MgCl}_{2}, 5 \mathrm{~mm}$ HEPES, pH 7.5. Single-channel recordings were made in response to step depolarizations from -90 to $0 \mathrm{mV}$ for $2 \mathrm{~s}$ with a $5 \mathrm{~s}$ interval between sweeps, and holding potential was $-90 \mathrm{mV}$. Data were sampled at $20 \mathrm{kHz}$ and filtered with analog Bessel filter at 5 kHz. Amplification was done with Axopatch 200B amplifier; acquired analog signal was digitized with DigiData 1200 B converter, recorded with Clampex 8.1, and analyzed in Clampfit 10.2 (pClamp; Molecular Devices). Event detection was performed using algorithm built-in pClamp10.2 software.

Single-channel data analysis. All events were checked visually before being accepted. The presence of only one channel in a patch was assumed if the opening of one channel exits in all sweeps until run down. After $\sim 30$ sweeps rundown of Po, usually of $>20 \%$, which did not recover during the following 10 sweeps, was observed and analysis was discontinued. Conductance levels were derived from amplitude histograms built up from pClamp10.2 generated event list. The histograms were fitted with a multicomponential Gaussian probability function, using Levenberg-Marquardt method (precision criterion for convergence was set to $10^{-6}$ ) with the sum of square errors minimization technique. Open probabilities (Po) were calculated separately from the event list as Po of independent events for each sweep until run down. First latencies [mean first latency (MFL)], calculated by constructing cumulative histograms and one-exponential rise function, were used to derive mean first latency values. 


\section{FRET analysis and confocal microscopy}

FRET experiments were performed as described previously (Lvov et al., 2009). Oocytes were injected with the following mRNA concentrations (in ng/oocyte): 45 double-labeled Q2, Q3* , or mutant channels, 0.75 Syx, or $5 \mathrm{CaM}$. Fluorescence emissions from eCFP- and/or eYFP-tagged proteins were collected from the animal hemisphere of the oocyte with a confocal microscope (Zeiss LSM 510 Meta), using a 20×, 0.75 NA objective and laser excitation of 405 and $514 \mathrm{~nm}$, respectively. The level of laser intensity and the photomultiplier tube gain were kept the same for all experiments after initial calibration and titration of proteins. We used spectrum-based method to remove contaminations caused by donor emission and direct excitation of the acceptor. The FRET assay was performed as described previously (Zheng and Zagotta, 2004). Briefly, two spectra were collected from each oocyte, one with $405 \mathrm{~nm}$ excitation and the other with $514 \mathrm{~nm}$ excitation. A scaled eCFP spectrum collected from control oocyte expressing eCFP-tagged channels were used to subtract eCFP emissions from the spectrum taken with $405 \mathrm{~nm}$ excitation, yielding an extracted eYFP emission spectrum, $F_{405} . F_{405}$ has two components, one due to direct excitation of eYFP, $F_{405}^{\text {direct }}$, and one due to FRET, $F_{405}^{\mathrm{FRET}} \cdot F_{405}$ is normalized to the total YFP emission with $514 \mathrm{~nm}$ excitation at the same oocyte, $F_{514}$. The resulted ratio, termed Ratio A, can be expressed as follows:

$$
\text { Ratio A }=\frac{F_{405}}{F_{514}}=\frac{F_{405}^{\text {direct }}}{F_{514}}+\frac{F_{405}^{\mathrm{FRET}}}{F_{514}} \text {. }
$$

The direct excitation component in the calculated Ratio A, termed Ratio $A_{0}$, is experimentally determined from a large population of oocytes expressing eYFP-tagged proteins only. This allows the precise calculations of the bleed-through or the contamination of the direct excitation of eYFP by the $405 \mathrm{~nm}$ laser. The difference between Ratio A and Ratio $\mathrm{A}_{0}$,

$$
\text { Ratio A - Ratio } \mathrm{A}_{0}=\frac{F_{405}^{\mathrm{FRET}}}{F_{514}},
$$

is directly proportional to FRET efficiency. The apparent FRET efficiency from individual cell, $E^{\text {app }}$ (FRET), can be calculated as follows:

$$
E^{\text {app (FRET) }}=\left(\frac{\text { Ratio } \mathrm{A}}{\text { Ratio } \mathrm{A}_{0}}-1\right) \frac{\varepsilon_{\mathrm{A}}}{\varepsilon_{\mathrm{D}}},
$$

in which $\varepsilon_{\mathrm{D}}$ and $\varepsilon_{\mathrm{A}}$ are molar extinction coefficients for the donor and acceptor, respectively, at the donor excitation wavelength (Takanishi et al., 2006; Gao et al., 2007).

For the control groups, Kv2.1 $\mathrm{C} 1 \mathrm{a}-\mathrm{Y}+\mathrm{C}$-syx and Kv2.1 $3331-\mathrm{Y}+$ $\mathrm{C}$-syx, data presented were extracted from eCFP/eYFP molar ratio $>3$ at which saturation of FRET occurred.

\section{Peripheral expression measurements by imaging}

Imaging C-Q2-Y peripheral channels with LSM 510 was performed as previously described (Regev et al., 2009). The thickness of the optical slide was $8 \mu \mathrm{m}$.

\section{Immunoprecipitation and immunoblotting in oocytes}

Oocytes were subjected to immunoprecipitation (IP) or immunoblotting (IB) analyses, as described previously (Levin et al., 1995; Regev et al., 2009).

\section{Expression and purification of the Q2 CT-CaM complex}

Transformed Escherichia coli BL-21 Tuner (Novagen), containing the "RIL" CodonPlus plasmid (Stratagene) were grown at $37^{\circ} \mathrm{C}$ in $\mathrm{LB}$ medium containing $100 \mu \mathrm{g} / \mathrm{ml}$ ampicillin and $34 \mu \mathrm{g} / \mathrm{ml}$ chloramphenicol. Upon reaching an $A_{600}$ of 0.3 , the temperature was lowered to $16^{\circ} \mathrm{C}$, and growth continued until the culture reached an $A_{600}$ of 0.6. Protein expression was induced with $135 \mu \mathrm{M}$ isopropyl 1-thio- $\beta$-D-galactopyranoside. Cells were harvested after $14 \mathrm{~h}$ by centrifugation and suspended in lysis buffer ( $300 \mathrm{~mm} \mathrm{NaCl}, 50 \mathrm{~mm}$ sodium phosphate, $\mathrm{pH} 8$, $1 \mathrm{~mm}$ phenylmethylsulfonyl fluoride). After lysis by a microfluidizer (Microfluidics), cell debris was removed by centrifugation at $30,600 \times g$. The soluble fraction was loaded onto a preequilibrated metal chelate $\mathrm{Ni}^{2+}$ -
NTA (QIAGEN) column (buffer A: $300 \mathrm{~mm} \mathrm{NaCl}, 50$ mm sodium phosphate, $\mathrm{pH} 8$ ) at a flow rate of $1.5 \mathrm{ml} / \mathrm{min}$. The column was washed with buffer A, containing $15 \mathrm{~mm}$ imidazole, until a stable base line was achieved. After elution with buffer A, supplemented with $250 \mathrm{~mm}$ imidazole, the fractions containing protein eluate were pooled and applied to a preequilibrated Superose 6 gel filtration column (GE Healthcare) with $200 \mathrm{~mm} \mathrm{NaCl}, 20 \mathrm{~mm}$ Tris, $\mathrm{pH}$ 7.5, and $1 \mathrm{~mm}$ dithiothreitol. The elution peak was concentrated using spin concentrators (Millipore) and flashfrozen in liquid $\mathrm{N}_{2}$.

In vitro pull-down of recombinant Q2CT-CaM complex with GST-fusion proteins of Q2 and Q3 NTs

This has been done as described previously (Jing et al., 1999). Briefly, purified GST-fusion proteins [GST-Q2NT or GST-Q3NT, 200 pmol; prepared as described by Regev et al. (2009)] were immobilized on glutathione-Sepharose beads and were incubated with 200 pmol of recombinant Q2 CT (residues 316-633 coexpressed with CaM, purified from E. coli) in $1 \mathrm{ml}$ of PBS containing a protein detergent $(0.1 \%$ Triton X-100). After washing, the GST fusion proteins were eluted with $20 \mathrm{~mm}$ reduced glutathione in $40 \mu \mathrm{l}$ of elution buffer, separated by $12 \%$ SDS-PAGE.

\section{Presentation and analysis of the experimental results}

Statistical estimations and graphical presentation of the data were performed in SigmaPlot and SigmaStat software (Systat Software). Comparisons between two groups were tested for statistical significance $(p<0.05$ or smaller) using two-tailed unpaired or paired $t$ tests according to the statistical problem requirement. Statistical evaluation of several groups comparison was performed using one-way ANOVA followed by Bonferroni's test. The data are presented as mean \pm SEM; $n$ denotes total number of oocytes per group; $N$ denotes number of experiments in different batches of oocytes.

Relative effects on current amplitudes reduction or FRET enhancement were calculated as the fraction decreased or enhanced, respectively, in response to Syx or CaM. Namely, in each test group of oocytes (channel coexpressed with Syx and/or CaM), current amplitude or FRET for each oocyte was divided by the mean value of the corresponding control group (expressing channel only) and subtracted from 1 . The obtained values were normalized to the corresponding mean value obtained for Q2. The data are presented as mean \pm SEM.

\section{Results}

\section{Syx decreases the Po of Q2 but not of Q3*}

Previously, we showed that Syx selectively affected Q2 but not Q3* a point mutation in the pore residue A315T introduced into Q3, as we could not detect currents above background levels in wild-type Q3 expressed in Xenopus oocytes, as was described by Etxeberria et al. (2004) (but see Schwake et al., 2000)], resulting in inhibition of macroscopic current amplitudes and slowing of activation kinetics, without any concomitant change in channel surface expression (Regev et al., 2009) (see Fig. 2B). This suggested that single Q2 but not Q3* channel characteristics, amplitude, and/or Po are affected by Syx. Here, we first tested this hypothesis analyzing single-channel behavior. Single-channel recordings in oocytes expressing Q2 or Q3* homomeric channels were performed using the cell-attached configuration of the patch-clamp technique. Figure $1 A$ shows representative traces of single Q2 (left) or Q3* (right) currents, expressed with or without Syx, at a saturating voltage of $0 \mathrm{mV}$. Whereas no effect of Syx on Q2 single-channel currents was detected (Fig. 1B,D), Q2 Po was significantly decreased by Syx (Fig. $1 E$ ). In contrast, Syx affected neither $\mathrm{Q}^{\star}$ single-channel currents (Fig. 1D) nor Q3* Po (Fig. $1 E)$. The Po and the single-channel current amplitude of Q2 were similar to the values reported in the literature $(0.11 \pm 0.01$ and $0.57 \pm 0.06$ pA, respectively; Selyanko et al., 2001; Li et al., 2004; Zaika et al., 2007). The Po of Q3* was somewhat smaller, whereas the current amplitude was similar to the reported values $(0.47 \pm$ 
A
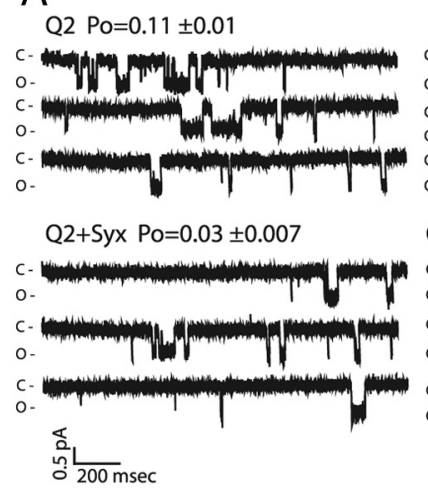

B
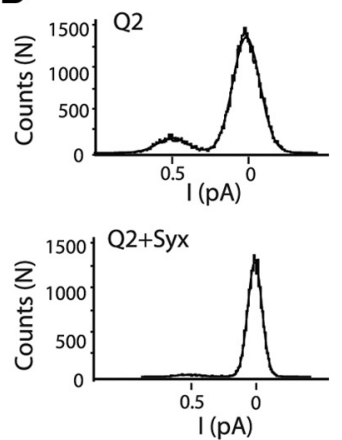

D

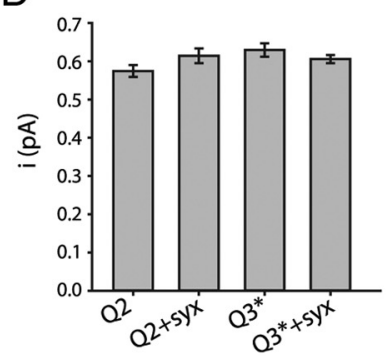

F

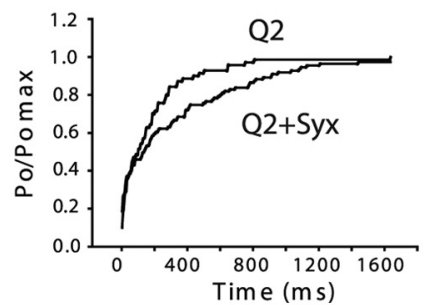

Q3* $\mathrm{Po}=0.47 \pm 0.03$

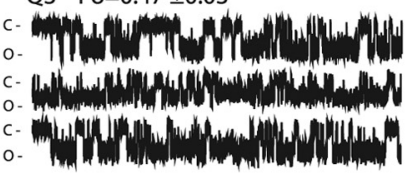

$\mathrm{Q}^{*}+$ Syx $\quad \mathrm{Po}=0.47 \pm 0.02$

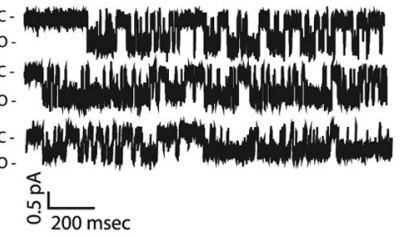

C
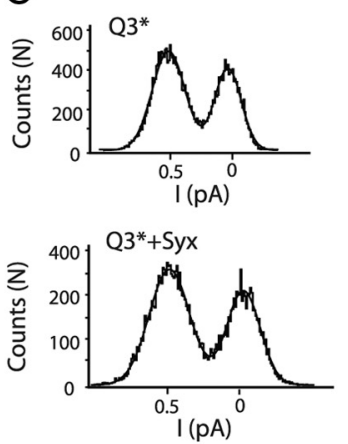

E

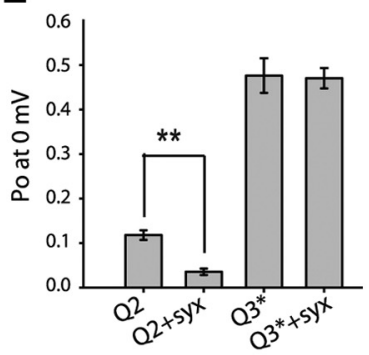

G

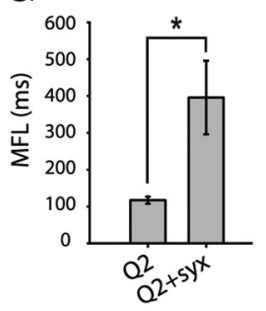

Figure 1. Syx decreases $\mathrm{P} 0$ of $Q 2$ but not $Q 3^{*}$. $\boldsymbol{A}$, Single-channel activity elicited by voltage steps from -90 to $0 \mathrm{mV}$ from 00 cyte patches containing a single Q2 or Q3* channel expressed alone or with Syx (+Syx). B, C, All-point amplitude histograms from the sweeps shown in $\boldsymbol{A}$ for Q2 and for $Q 3^{*}$, respectively, alone or with Syx, fitted by a double-Gaussian curves (smooth lines). The closed-state peak has been set to 0 pA. D, Mean amplitudes ( $i$ ) of Q2 and Q3* , alone or coexpressed with Syx ( $n=9-16$ patches). $\boldsymbol{E}$, Po values of $Q 2$ and $Q 3^{*}$ averaged from patches containing one to three channels, alone or coexpressed with Syx ( $n=9-16$ patches per group). $\boldsymbol{F}$, Normalized Po obtained from cumulative histograms of first latency (with $5 \mathrm{~ms}$ bins) derived from analysis of $>70$ sweeps from $Q 2$ and $\sim 140$ sweeps from 02 coexpressed with Syx. G, MFL time values; equal number of sweeps as in $\boldsymbol{F} .{ }^{*} p<0.05$; ${ }^{* *} p<0.01$. Error bars indicate SEM.

0.03 and $0.62 \pm 0.01 \mathrm{pA}$, respectively; Zaika et al., 2007). The discrepancy between the Q $3{ }^{\star}$ Po values may be accounted for by the different expression systems. Together, we conclude that a decrease in Po underlies the effect of Syx to reduce the macroscopic currents of Q2. Namely, the interaction of Syx with Q2

downregulates channel gating. In addition, kinetic analysis of MFL values of Q2 derived from first latency cumulative histograms, obtained in membrane patches of oocytes expressing Q2, alone or together with Syx, showed significantly lower values in the presence of Syx (Fig. $1 F, G$ ). This attenuation of single Q2 channel rate of activation by Syx is consistent with our previous results describing an attenuation of the rate of activation of macroscopic Q2 current by Syx (Regev et al., 2009), vouching for the reliability of the single-channel analysis.

The selective effect of Syx on Q2 but not on Q3* is not due to different Syx binding capacities

Our next aim was to map the structural domains involved in the selective effect of Syx. Previously (Regev et al., 2009), a strong in vitro binding of Syx to the Q2 C-terminal helix A and inability of Syx to associate with Q2 lacking helix A in oocytes gave support to the notion that helix A was a crucial region for Syx association with Q2 homomeric channels. In contrast, only weak association of homomeric Q3 channels with Syx was detected and assumed to be due to the weaker Syx binding to Q3 helix A, despite the high homology shared with Q2 helix A. Here, to validate the assumption, we exchanged helices A between Q2 and Q3, generating the chimeras $\mathrm{Q}_{\left(\mathrm{Q}^{*}\right.}$ helixA) and $\mathrm{Q}_{(\mathrm{Q} 2 \text { helixA })}^{*}$, and performed twoelectrode voltage-clamp (TEVC) analysis of the macroscopic currents of the WT and the chimeric channels. Unexpectedly, Syx reduced $\mathrm{Q}_{\left(\mathrm{Q}^{*}\right.}$ helixA) and did not significantly affect $\mathrm{Q} 3_{(\mathrm{Q} 2}^{*}$ helixA) current amplitudes, similarly to its effects on the corresponding WT channels (Fig. 2A-D). Also, the association of Syx with the chimeric channels assayed in coimmunoprecipitation (co-IP) experiments, using antibodies against Q2 and Q3, were similar to its association with the corresponding WT channels (data not shown). These findings were puzzling in light of the selective functional interaction of Syx with Q2 versus Q3. To approach this problem, we revisited the co-IP experiments, now using a single antibody, common for both channels, whereas formerly different antibodies, specific for Q2 or for Q3, were used (Regev et al., 2009). Notably, similar amounts of Syx were coimmunoprecipitated by HA-tagged Q2 and Q3, using anti-HA antibody (Fig. $2 E)$. Thus, while Syx binds similarly to Q2 and Q3 channels, presumably at their helix A domains (Regev et al., 2009), its subunit-specific function on channel activity is mediated by channel region(s) other than helix A.

The effects of Syx on current amplitudes of Q2 and Q3* are dependent on $\mathrm{N}$-terminal distal-end parts of the channels Next, we aimed to map the domains responsible for the function of Syx and tested the effect of Syx on current amplitudes of a set of chimeras, in which whole or parts of the NT or CT were exchanged between Q2 and Q3* (Fig. 3A). The effect of Syx was retained in the chimera $\mathrm{Q} 2_{\mathrm{N}} / \mathrm{Q} 3_{\mathrm{C}}^{*}$, in which the $\mathrm{CT}$ of $\mathrm{Q} 2$ was exchanged with that of $\mathrm{Q}^{*}$, and was abolished in $\mathrm{Q} 3{ }_{\mathrm{N}}^{*} / \mathrm{Q} 2_{\mathrm{C}}$, in which the NT of Q2 was exchanged with that of Q3* (Fig. 3B). These findings suggested that structural differences exist between the NTs of Q2 and Q3, enabling Syx function in Q2 and preventing it in $\mathrm{Q}^{*}$.

Next, we aimed to map down the NT structural differences, using Syx effect as a probe. Toward this end, we constructed several chimeric channels with different NT parts swapped between Q2 and Q3* (Fig. 3A) and investigated the effect of coex-

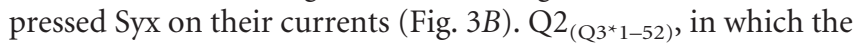
distal 52 aa of the NT of Q2 were exchanged with those of Q3, showed loss of Syx function (Fig. 3B, left panel). Furthermore, alignment of Q2 and Q3 showed a 14 aa sequence, comprising a 
glycine-rich motif (GR motif) in the distal end of Q3 NT, which does not exist in Q2 (Fig. 3A, bottom panel). Analysis of the effect of Syx on Q2 ${ }_{\left(\mathrm{Q}^{*} 7-20\right)}$, in which the GR motif (amino acids 7-20) from Q3* was inserted between amino acids 6 and 7 of Q2, showed loss of Syx function (Fig. $3 B$, left panel). Remarkably, reciprocal analysis of the effect of Syx in Q3 ${ }_{(\Delta 5-26)}^{*}$ a Q3* channel with deletion of a 21 aa stretch (amino acids 5-26) that includes the GR motif, showed gain of Syx function (Fig. 3B, right panel). Namely, the insertion of the GR motif to Q2 rendered the channel nonresponsive to Syx, as is the case for $\mathrm{Q}^{*}$, and its deletion from $\mathrm{Q}^{*}$ conferred the response to Syx, as observed in Q2. As glycine residues are known to bestow flexibility to protein structures ( $\mathrm{Li}$ and Deber, 1992; Bright and Sansom, 2003), we reasoned that the GR motif may bestow flexibility to an upstream NT distal-end determinant, enabling its interaction with the CT. Thus, we generated a Q3* construct, $3^{*}{ }_{(\Delta 1-6)}^{*}$, which carries a deletion of the first 6 a from the distal end of the NT that precede the GR motif. As in the case of $\mathrm{Q}_{(\Delta 5-26)}^{*}$, coexpression of Syx with Q3 ${ }_{(\Delta 1-6)}^{*}$ reduced the current amplitudes to the same extent as Q2 (Fig. $3 B$, right panel). Concomitant co-IP analysis in oocytes coexpressing HA-tagged channels and Syx, using anti-HA antibody, confirmed that, as expected, both Q2 and Q3, as well as two selected chimeric channels, $2_{\left(\mathrm{Q}^{*} 7-20\right)}$ and $\mathrm{Q} 2_{\left(\mathrm{Q}^{*}{ }^{*}-52\right)}$, all bound Syx to a similar extent (Fig. 3C), substantiating our previous conclusion that different bindings of Syx to the channels do not underlie the selective effect of Syx.

Together, the TEVC and biochemical analyses of the interactions of the different chimeric channels with Syx, localized the selective effect of Syx to the NT distal-end module, specific for Q3, which is composed of the GR motif and the distal-end determinant. Of note, GR motif inserted to Q2 NT ( $\left.2_{\left(\mathrm{O}^{*} 7-20\right)}\right)$ probably bestows flexibility to the first 6 aa of Q2, together forming an NT distal-end module similar to that of Q3, which hampers the effect of Syx on Q2. Namely, Q2 bears a truncated NT distal-end module.

\section{NT distal-end module of $\mathrm{Q}^{*}$ prevents the effect of Syx on unitary Po}

To substantiate the role of the NT distal-end module, we performed single-channel patch-clamp analysis (as in Fig. 1) on three chimeras, one that lost and two that gained the effect of Syx on macroscopic current amplitudes (Fig. 3B), alone or coexpressed with Syx (Fig. 4). The Po of chimera Q2 ${ }_{\left(\mathrm{Q} 3^{*} 7-20\right)}$, in which insertion of the GR motif from Q3 NT into Q2 NT caused loss of Syx function, was not affected by Syx (Fig. $4 A, B$ ). However, Syx sig-
B

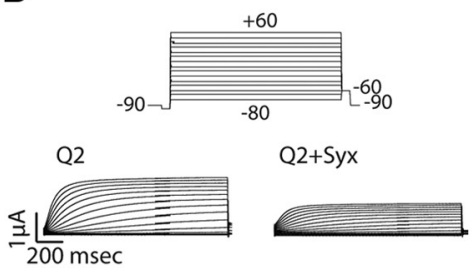

Q3*
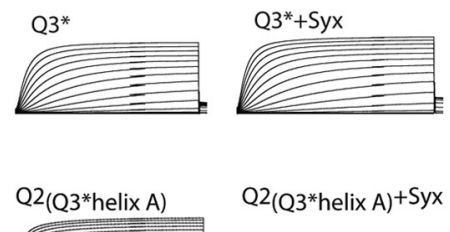

Q2(Q3*helix A)+Syx
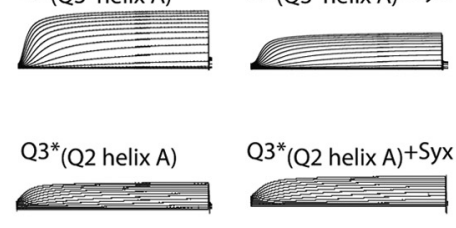

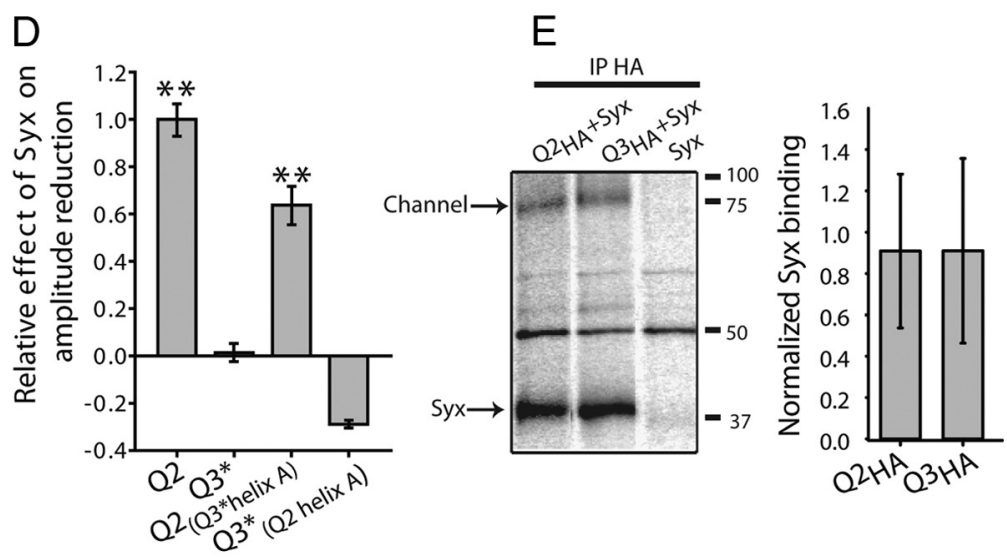

Figure 2. The selective effect of Syx on Q2 but not on Q3* current amplitude is not due to different Syx binding capacities. $A$, chematic representations of Q2 (white) and Q3* (black) and a pair of chimeras with exchanged helix A domains. $\boldsymbol{B}$, Representative 政 rmalized averaged current amplitudes, evoked by a voltage step from a holding potential of -90 to $+5 \mathrm{mV}$, of the WT and ic channels, alone or coexpressed with Syx $(n=25-35 ; N=4)$. $\boldsymbol{D}$, Relative effect of Syx on current amplitudes, quantified (a)

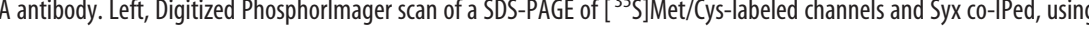
anti-HA antibody (IP HA), from homogenates of oocytes of a single batch expressing $\mathrm{Q2}_{\mathrm{HA}}, \mathrm{Q3}_{\mathrm{HA}}^{*}$ Syx, or both, as denoted above the lanes. Right, Normalized Syx binding calculated as band intensity ratio of Syx to channel (arbitrary units quantitated by ImageQuant; $N=3) .{ }^{* *} p<0.01$. Error bars indicate SEM.

nificantly reduced the Po values of the chimeras $\mathrm{Q}_{(\Delta 5-26)}^{*}$ (Fig. $4 C, D)$ and $\mathrm{Q} 3{ }_{(\Delta 1-6)}^{\star}$ (Fig. $\left.4 E, F\right)$, in which truncations of the Q3 NT distal-end module caused gain of Syx function. Thus, the effects of Syx on Po correlated with those on macroscopic currents (Fig. 3B) and supported a role for the NT distal-end parts of the channels in the Syx modulation. Of note, the insertion in Q2 or the deletions in $\mathrm{Q}^{*}$ did not significantly affect the Po values of the channels expressed alone.

\section{Different $\mathrm{N}-\mathrm{C}$ interactions in Q2 and Q3*; the difference is conveyed by the Q3 NT distal-end module}

The above results suggested a possible cross talk between closely positioned $\mathrm{N}$ and $\mathrm{C}$ termini. We used the FRET approach to 


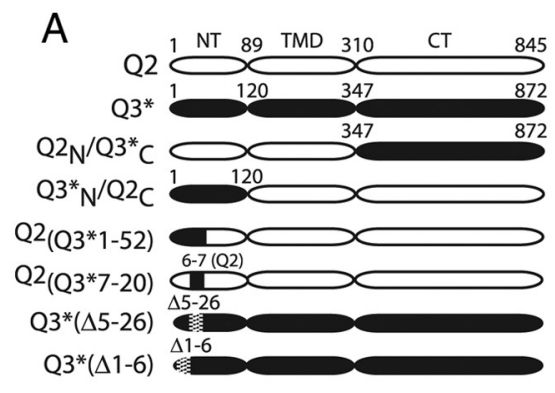

Q2 1 MVQKSR--------------NGGV 9

Q3 1 MGLKARRAAGAAGGGGDGGGGGGG 23
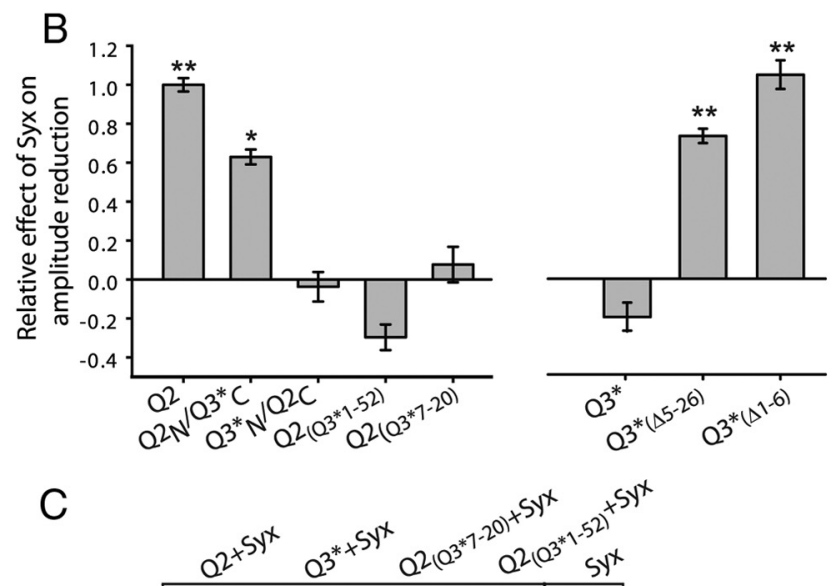

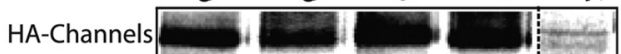

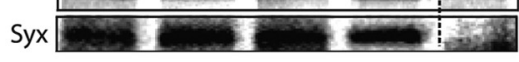

IP HA

Figure 3. The effects of Syx on current amplitude of $Q 2$ and $Q 3^{*}$ are dependent on the NT distal-end parts of the channels. A, Top panel, Schematic representation of Q2 (white) and Q3* (black) chimeras. $Q 2_{\left(\mathrm{Q}^{*} 7-20\right)}$, Glycine-rich motif (amino acids 7-20) of $\mathrm{Q3}$ inserted between amino acids $6-7$ of $Q 2 ; 03_{(\Delta 5-26)}^{*}, 21$ aa $(5-26)$ deleted from $Q 3^{*}$ (checkered); $Q 3_{(\Delta 1-6)}^{*}$ first 6 aa deleted from Q3* ${ }^{*}$. Bottom panel, Alignment of the NT distal-end parts of Q2 and Q3 shows a glycine-rich 14 aa stretch in Q3 that is missing in Q2. Alignment was performed using CLUSTAL W (1.83) multiple sequence alignment. $\boldsymbol{B}$, Left, Loss of Syx function to reduce current amplitude is dependent on changes in Q2 NT distal-end part, and not its CT. Right, Gain of Syx function to reduce current amplitude in $\mathbf{Q 3}^{*}$ is dependent on changes in $\mathrm{Q3}$ NT distal-end parts. Shown are relative effects of Syx (quantified as in Fig. 2D) on the different chimeras shown in $A(n=9-57$; $N=1-5)$. C, Syx coprecipitates equally well with all HA-tagged Q2 and chimeric channels from oocytes coexpressing the channels with Syx, using anti-HA antibody, performed as in Figure $2 E$. ${ }^{*} p<0.05 ;{ }^{* *} p<0.01$. Error bars indicate SEM.

monitor the $\mathrm{N}-\mathrm{C}$ proximity in functional intact channels under physiological conditions. Cross talk was assessed by the apparent FRET efficiency (FRET) between eCFP (C) and eYFP (Y) (as done previously; Lvov et al., 2009), genetically attached to the distal ends of NTs and CTs, respectively, of Q2, Q3*, and the chimeric channels. The double-labeled channels retained WT voltage sensitivity [Fig. 5A, see for double-labeled Q2 (C-Q2-Y) and $\left.\mathrm{Q}^{*}\left(\mathrm{C}-\mathrm{Q} 3^{*}-\mathrm{Y}\right)\right]$ and response to Syx (Fig. 6D). However, to get current amplitudes similar to WT, the mRNA concentrations were increased by $\sim 25 \%$. Representative confocal images of oocytes expressing the labeled channels that were taken under 405 and $514 \mathrm{~nm}$ laser excitations and the emission spectra obtained under $405 \mathrm{~nm}$ laser excitation are shown in Figure 5, $B$ and $C$, respectively. Oocytes expressing C-Q2-Y or C-Q3*-Y channels exhibited FRETs between their $\mathrm{N}$ and $\mathrm{C}$ termini, that of $\mathrm{C}-\mathrm{Q} 3^{*}-\mathrm{Y}$ being higher (Fig. 5C-E). Although being small, the difference
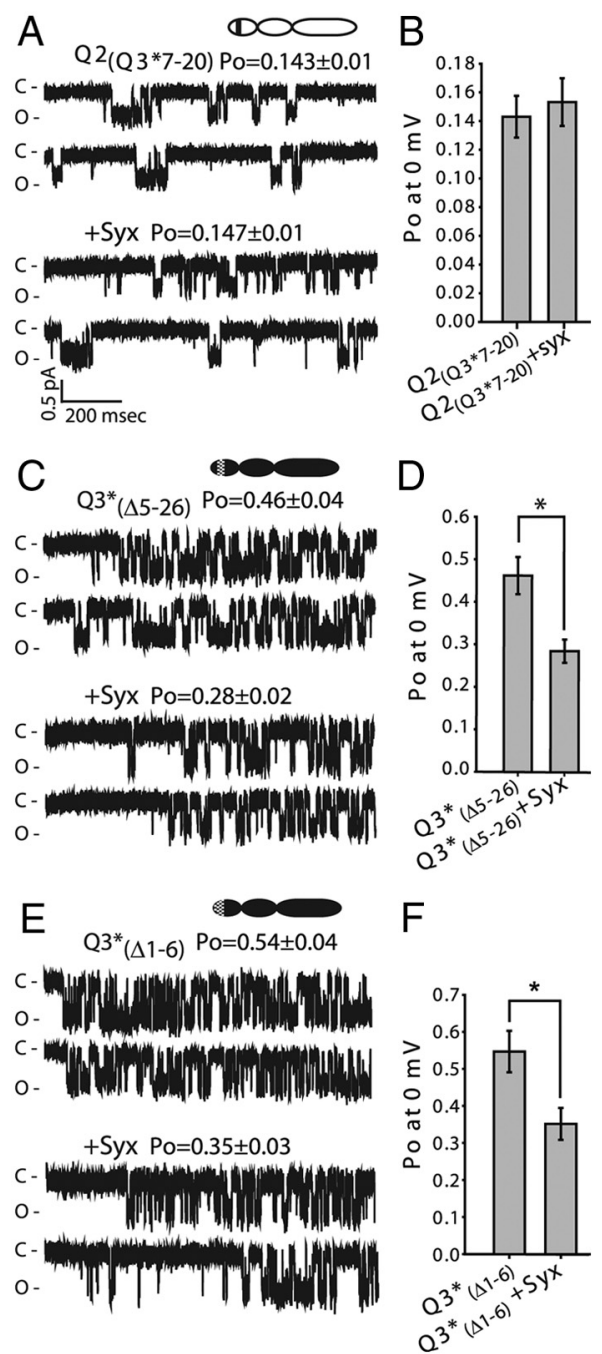

Figure 4. NT distal-end parts control the effect of Syx on Po. A, C, E, Single-channel recordings performed as in Figure 1 from oocyte patches containing single $Q_{\left({ }_{\left(03^{*} 7-20\right)}\right.}(A), Q 3_{(\Delta 5-26)}^{*}$ $(\boldsymbol{C})$, or Q3 ${ }_{(\Delta 1-6)}^{*}(\boldsymbol{E})$ channels, alone or together with Syx (+Syx). B, D, F, Corresponding Po values averaged from patches containing one to three channels $(n=5-8) .{ }^{*} p<0.05$. Error bars indicate SEM.

between the FRETs of the two channels was statistically significant and was persistent in each one of the nine similar experiments performed. Serving as a positive control was the FRET between coexpressed C-Syx (eCFP fused to the NT of Syx) and the Kv2.1 channel mutant, Kv2.1 $1331-\mathrm{Y}$ (in which eYFP is fused next to the Syx binding domain, C1a; Fig. 5D). This Kv2.1 mutant has been shown before to bind Syx (Michaelevski et al., 2003). As a negative control, we used oocytes coinjected with C-Syx and Kv2.1 $\Delta$ C1a-Y (eYFP fused to the CT of a mutant Kv2.1 channel that carries a deletion of C1a), which exhibited no FRET (Fig. $5 C, D$ ), as expected (Michaelevski et al., 2003). This set of experiments established a close proximity between the termini, which differ between Q2 and Q3* channels.

Next, we asked whether the difference between the N-C interactions of Q2 and Q3* is conferred by the Q3 NT distalend module. The FRET of $\mathrm{C}-\mathrm{Q} 2_{\left(\mathrm{Q} 3^{*} 7-20\right)^{-}} \mathrm{Y}$ was not significantly different from that of $\mathrm{C}-\mathrm{Q} 3^{*}-\mathrm{Y}$, both bearing the GR motif. However, significantly lower FRET, similar to that of C-Q2-Y, was observed in C-Q3 ${ }_{(\Delta 1-6)}^{\star}$ Y with a truncated NT distal-end determinant (Fig. $5 D, E$ ). Hence, it was postulated that the Q3 NT distal-end module strengthens the N-C direct 

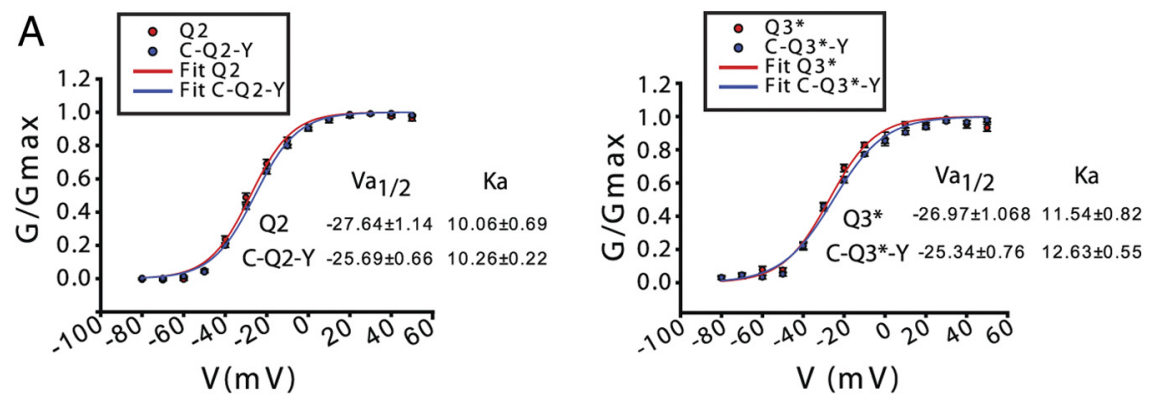

B

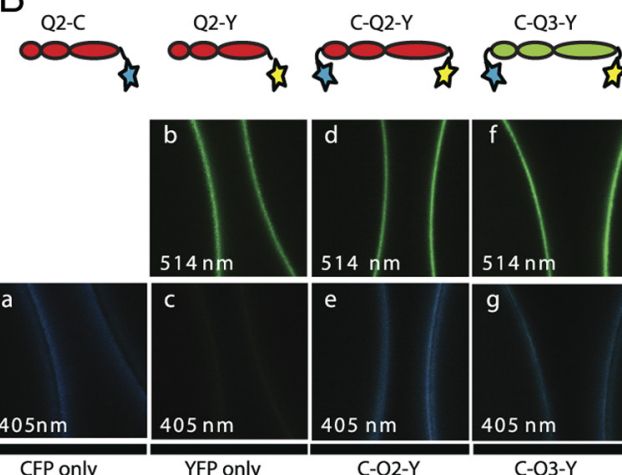

C-Syx+ $\mathrm{Kv} 2.1 \Delta \mathrm{C} 1 \mathrm{a}-\mathrm{Y} \quad \mathrm{C}-\mathrm{Q} 3^{*}(\Delta 1-6)^{-\mathrm{Y}} \quad \mathrm{C}-\mathrm{Q} 2{ }^{2}\left(\mathrm{Q} 3^{*} 7-20\right)^{-\mathrm{Y}}$
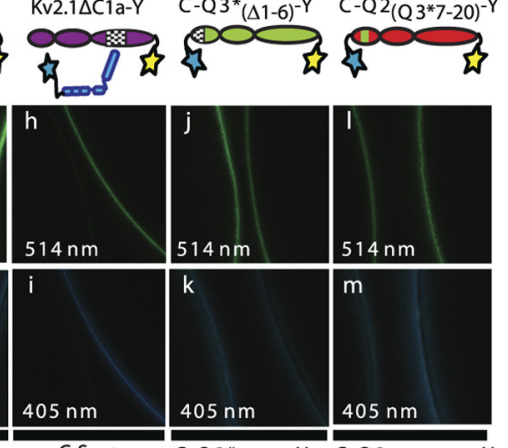

C-Syx+ $\mathrm{Kv} 2.1 \Delta \mathrm{C} 1 \mathrm{a}-\mathrm{Y}$
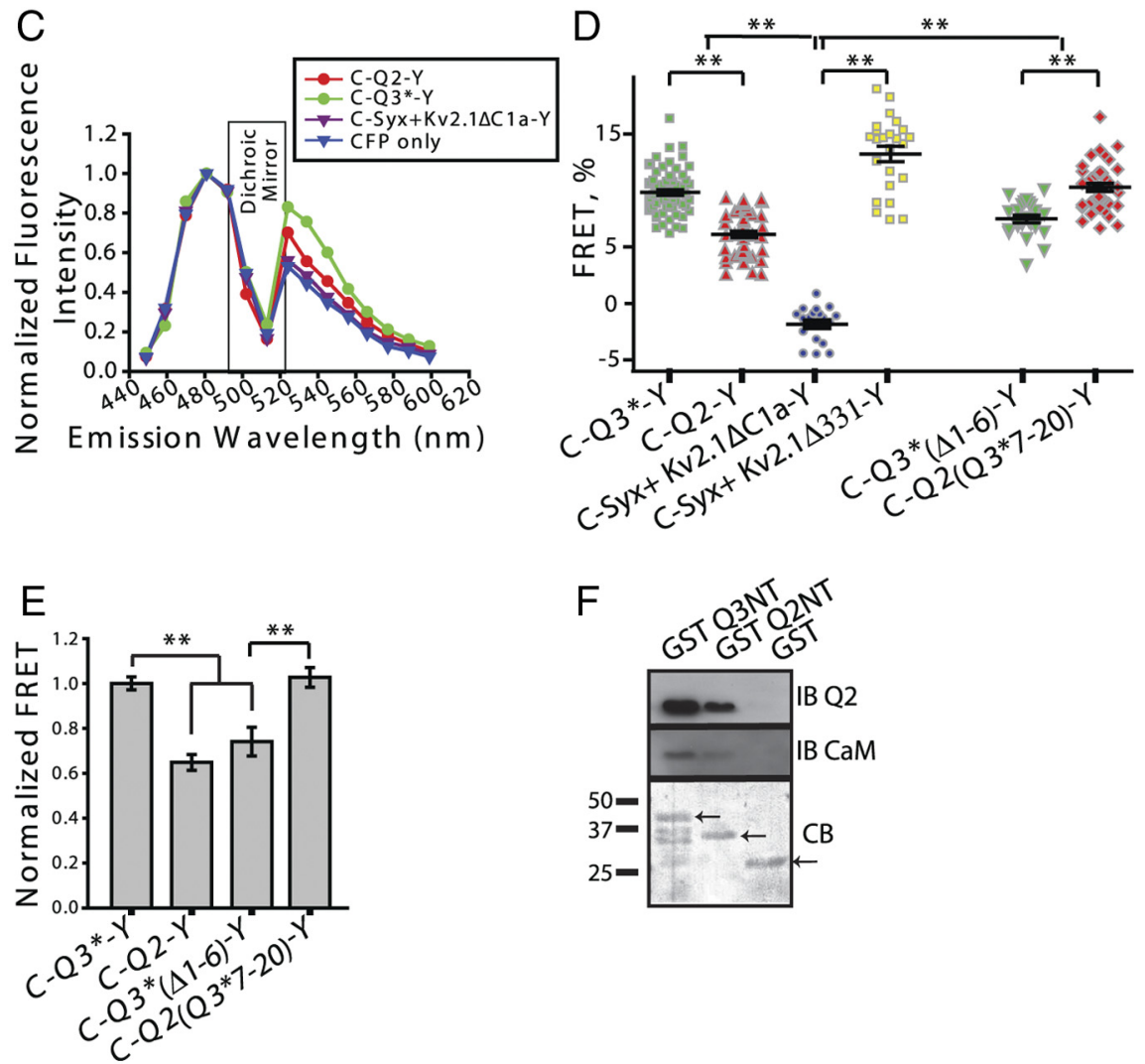

Figure 5. FRET and biochemical analyses demonstrate $\mathrm{N}-\mathrm{C}$ termini direct interactions in $\mathrm{Q2}$ and $\mathrm{Q3}$ *; the interactions are different and are regulated by NT distal-end parts. $\boldsymbol{A}$, Double fluorescence labeling of $\mathrm{Q} 2$ and $Q 3^{*}$ does not impair voltage sensitivity. 0ocytes were injected with WT (red) Q2 (left) or Q3*(right) mRNAs or double fluorescent-labeled channels (blue) C-Q2-Y (left) or $C-Q 3^{*}-Y$ (right) mRNAs. Normalized conductance $\left(G / G_{\max }\right)$-voltage ( $V$ relationships were derived from current-voltage relationships obtained from leak subtracted peak currents elicited by $1.5 \mathrm{~s}$ steps to the denoted potential (with $5 \mathrm{~s}$ intervals between episodes); half-activation voltages $\left(\mathrm{Va}_{1 / 2}\right)$ and activation slope factors $(\mathrm{Ka})$ derived from exponential curve fitting (Fit) of WT and fluorescent labeled channels are denoted. $N=2 ; n=12-18 . B$, Confocal images of two representative oocytes in a frame expressing the different fluorescent-labeled channels [denoted above the images with eCFP (blue star) and/or eYFP (yellow star) decorations] excited with $405 \mathrm{~nm}$ or with $514 \mathrm{~nm}$ laser excitations. Note the very weak emission of Q2-eYFP (Q2-Y) caused by $405 \mathrm{~nm}$ excitation (c). The lighter blue shade of image $\boldsymbol{e}$, in comparison with image $\boldsymbol{a}$, and even lighter blue shade of image $\boldsymbol{g}$ in interaction in Q3, on top of a basal interaction that exists in both Q2 and Q3 ${ }^{*}$.

We next set out to assess whether the $\mathrm{N}-\mathrm{C}$ close proximity monitored by the FRET analysis reflects physical interactions between the termini. To this end, we conducted an in vitro pull-down assay, using immobilized NTs of Q2 or Q3 fused to glutathione $S$-transferase (GST-Q2NT and GST-Q3NT) and a purified recombinant Q2 CT complex beginning at residue 316 and extending to residue 633. This Q2 $\mathrm{CT}$ complex is oligomeric and has CaM associated with it, in a parallel manner as a recombinant KCNQ1 CT/CaM complex preparation, previously described (Wiener et al., 2008). Both NTs bound the Q2 CT complex. The binding to Q3 NT was significantly stronger (Fig. $5 F$ ), bearing resemblance to the FRET results showing stronger $\mathrm{N}-\mathrm{C}$ interaction in $\mathrm{Q} 3$ than in Q2 (Fig. 5D,E). Together, we detect direct and physical interactions between the termini of Q2 and Q3, where Q3 NT may bind more avidly.

\section{Syx differently affects the $\mathrm{N}-\mathrm{C}$ direct interactions of $\mathrm{Q} 2$ and $\mathrm{Q} 3^{*}$; the difference is conveyed by the Q3 NT distal-end module}

Channel gating modulators have been shown to alter the relative interactions between intracellular channel domains (Lippiat et al., 2002; Tsuboi et al., 2004; Lvov et al., 2009). Thus, we set out to investigate whether the Q2 gating downregulation by Syx is accompanied by

comparison with images $\boldsymbol{a}$ and $\boldsymbol{e}$, are indicative of FRET. $\boldsymbol{c}$ Emission spectra of eCFP only (blue), $C-02-Y$ (red), $C-03^{*}-Y$ (green), or C-Syx (eCFP inserted at the N terminus of Syx) with Kv2.1DC1a-Y (a Kv2.1 channel mutant with deleted C1a domain with eYFP inserted at its ( terminus; purple). Oocytes were excited at $405 \mathrm{~nm}$ and the resulting spectra were normalized to the peak eCFP emission ( $481 \mathrm{~nm}$ ). D, FRET between $\mathrm{N}$ and $\mathrm{C}$ termini of $\mathrm{C}-\mathrm{Q2}-\mathrm{Y}, \mathrm{C}-\mathrm{Q} 3^{*}-\mathrm{Y}$, and the chimeras $\mathrm{C}-\mathrm{Q2} 2_{\left(03^{*} 7-20\right)^{-}}-\mathrm{Y}$ and $C-Q 3_{(\Delta 1-6)}^{*}-Y$, in which the GR motif was exchanged. FRET between C-Syx and Kv2.1 $\Delta 331-Y$ (a Kv2.1 channel mutant with eYFP inserted downstream to the C-terminal C1a domain, which binds Syx) serves as a positive control. No significant FRET between C-Syx and Kv2.1 $\Delta$ C1a-Y (a Kv2.1 mutant with deleted (1a domain and eYFP inserted at its ( terminus) serves as a negative control. The results shown are pooled from two to nine similar independent experiments $(n=19-60)$. $\boldsymbol{E}$, Averaged FRETs (derived from the data presented in $\boldsymbol{D}$ ) normalized to that of $\mathrm{C}-\mathrm{Q} 3^{*}-\mathrm{Y}$. $\boldsymbol{F}$, Direct $\mathrm{N}-\mathrm{C}$ termini interaction demonstrated by Western blot analysis of $\mathrm{Q} 2$ recombinant $\mathrm{CT}$ (synthesized with (aM) pulled down by Q2 or Q3 GST-NTs. Eluted proteins were separated by SDS-PAGE and either immunoblotted (IB) with anti-Q2 or anti-CaM antibodies to show the bound proteins (top panels) or stained with Coomassie Blue (CB) to show the GST proteins (bottom panel). ${ }^{* *} p<$ 0.01 . Error bars indicate SEM. 

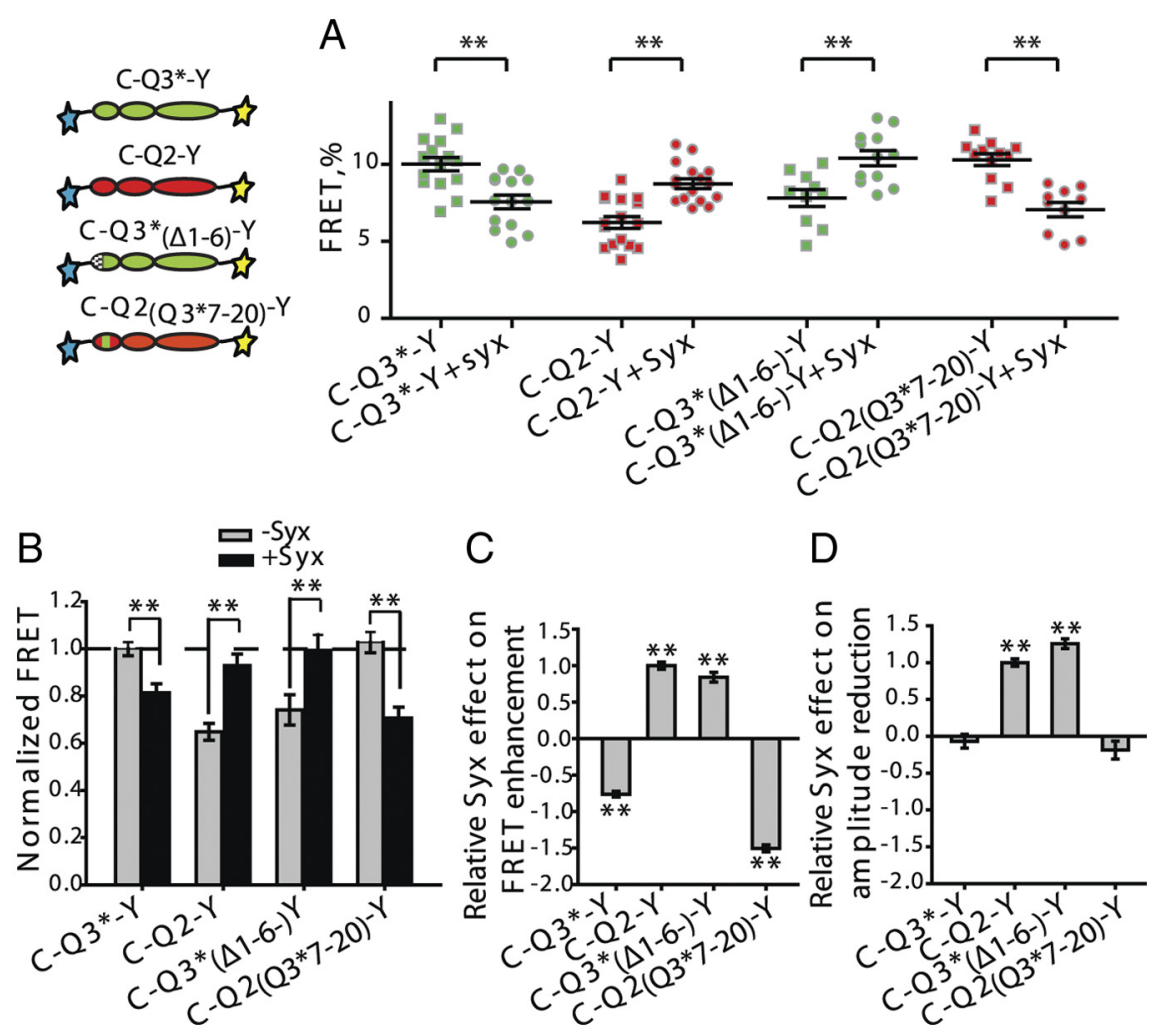

Figure 6. Syx affects the N-CFRET of double fluorescent-labeled Q2 and Q3*; the effects are different and are determined by NT distal-end parts, similarly to the effects on the corresponding current amplitudes. $\boldsymbol{A}, \boldsymbol{B}$, Syx reduces the FRET in $C-Q 3^{*}-\mathrm{Y}$ and increases that of $C-Q 2-Y$; changes in the NTs $\left(C-Q 2_{\left(Q 3^{*} 7-20\right)}-Y\right.$ and $\left.C-Q 3_{(\Delta 1-6)}^{*}-Y\right)$ reverse the effects of Syx. Oocytes were injected with the mRNAs of each of the channels, alone or with Syx. Shown are FRETs of one representative experiment $(n=10-16 ; \boldsymbol{A})$ and averaged FRETs, normalized to that of $C-Q 3^{*}-Y$, pooled from one to three similar experiments $(n=6-38 ; \boldsymbol{B})$. C, D, Relative effect of Syx on FRET enhancement (quantified as the fraction of FRET enhanced in the presence of Syx, normalized to fraction enhanced by Syx in Q2; $C$ ) and on current amplitude reduction (quantified as the fraction of current amplitude reduced in the presence of Syx, normalized to the fraction reduced by Syx in $Q 2 ; D)$, assayed in the same 00 cytes. Note the correlation between the relative effects of Syx in $\boldsymbol{C}$ and $\boldsymbol{D}$. ${ }^{* *} p<0.01$. Error bars indicate SEM.

specific changes in the $\mathrm{N}-\mathrm{C}$ interactions. We were encouraged by the findings that the gating downregulation by Syx (Figs. 3, 4) was dependent on the NT distal-end module, which was also implicated in the $\mathrm{N}-\mathrm{C}$ interactions (Fig. 5D,E). Upon coexpression with Syx, FRETs of C-Q2-Y and of C-Q $3_{(\Delta 1-6)}^{*}$ Y with truncated NT distal-end module significantly increased, whereas FRETs of $\mathrm{C}-\mathrm{Q} 3^{*}-\mathrm{Y}$ and $\mathrm{C}-\mathrm{Q} 2_{\left(\mathrm{Q}^{*} 7-20\right)}-\mathrm{Y}$, both channels bearing intact $\mathrm{NT}$ distal-end module, significantly decreased (a representative experiment and data pooled from several experiments are shown in Fig. $6 A, B$, respectively). Importantly, the Syx-induced increases of FRET clearly correlated with the Syx-induced reductions of current amplitude, whereas the Syx-induced reductions of FRET correlated with failure of Syx to reduce current amplitudes (Fig. 6 , compare $C, D$; measured in the same oocytes). Of note, the "Syx-responding" (electrophysiologically wise) channels exhibited smaller initial FRET in the absence of Syx, which was increased by coexpressed Syx to about the initial FRET level of the "nonresponding" channels (Fig. 6B, dotted line). Together, the electrophysiological and the FRET analyses suggest that Syx affects the relative orientation of the $\mathrm{N}$ and $\mathrm{C}$ termini, subject to their initial orientation, which is influenced by the NT distal-end module. Furthermore, increased FRET accompanies gating downregulation by Syx. Namely, the initial mode of the $\mathrm{N}-\mathrm{C}$ interaction, dictated by the NT distal-end module, determines the Syx-induced changes in the $\mathrm{N}-\mathrm{C}$ interaction and gating.
CaM decreases the current amplitudes and increases the $\mathrm{N}-\mathrm{C}$ interactions of Q2 but not of $\mathrm{Q}^{*}$; subunit selectivity is conferred by the CTs

$\mathrm{Ca}^{2+}$-CaM function had been previously suggested to reduce Q2 but not Q3* currents in sympathetic superior cervical ganglion neurons and in $\mathrm{CHO}$ cells (Gamper et al., 2005). However, there are still some discrepancies concerning the $\mathrm{Ca}^{2+}$ dependence of CaM modulation and whether it is an auxiliary subunit that enhances channel surface expression and/or a modulator (Wen and Levitan, 2002; Gamper and Shapiro, 2003; Gamper et al., 2005; Bal et al., 2008; Etxeberria et al., 2008). Here, we set out to investigate the regulation of Q2 by CaM in oocytes. Since the site of CaM binding (helices A and B; Wen and Levitan, 2002; YusNajera et al., 2002; Gamper and Shapiro, 2003) somewhat overlaps with that of Syx (helix A; Yus-Najera et al., 2002; Regev et al., 2009) and both proteins selectively reduce Q2 current amplitudes, we wondered whether the two regulatory proteins also share the same mechanism of action.

Initial experiments with different concentrations of CaM or $\mathrm{CaM}_{1234}$ (in which all four EF hands have aspartate-toalanine mutation, rendering it unable to bind $\mathrm{Ca}^{2+}$; Geiser et al., 1991) coexpressed with a standard Q2 concentration (same concentration was used for the experiments with Syx) showed no significant effects on current amplitudes (Fig. $7 A$ ). Also, further manipulations (as has been done in $\mathrm{CHO}$ cells to demonstrate Q2 current amplitude reduction by CaM; Gamper and Shapiro, 2003) failed to recapitulate the effect of CaM in oocytes (data not shown). We reasoned that, at standard levels of Q2 expression, the high level of endogenous CaM in oocytes (34 $\mu \mathrm{M} \mathrm{CaM/}$ oocyte; Cartaud et al., 1980) saturates the Q2 channels and only at higher level of expression of Q2, which will reduce the endogenous CaM/channel ratio, that the effect of overexpressed CaM on the channel will be manifested. Indeed, in the following experiments, CaM coexpressed with higher expression levels of the channels did reduce the Q2 but not the Q3* currents; the extent of reduction was small but statistically significant (Fig. $7 B$ ). The reduction of currents was not accompanied by reduction of channel peripheral expression, determined by confocal imaging of C-Q2-Y expressed alone or together with CaM (Fig. 7C). Importantly, peripheral measurements by confocal imaging were previously shown to highly correlate with surface expression of Q2 (Regev et al., 2009) and other channels (Rubinstein et al., 2009). Together, this suggests that overexpressed CaM downregulates Q2 channel gating. Also, $\mathrm{CaM}_{1234}$ coexpressed with high levels of Q2 reduced Q2 currents (Fig. 7B); however, this effect was accompanied by a similar extent of reduction in channel peripheral expression (Fig. 7C; in agreement with the suggested role for $\mathrm{CaM}_{1234}$ in Q2 retention to the ER; Etxeberria et al., 2008), suggesting that $\mathrm{Ca}^{2+}$-binding capacity of $\mathrm{CaM}$ is needed for the downregulation of Q2 gating. 
A
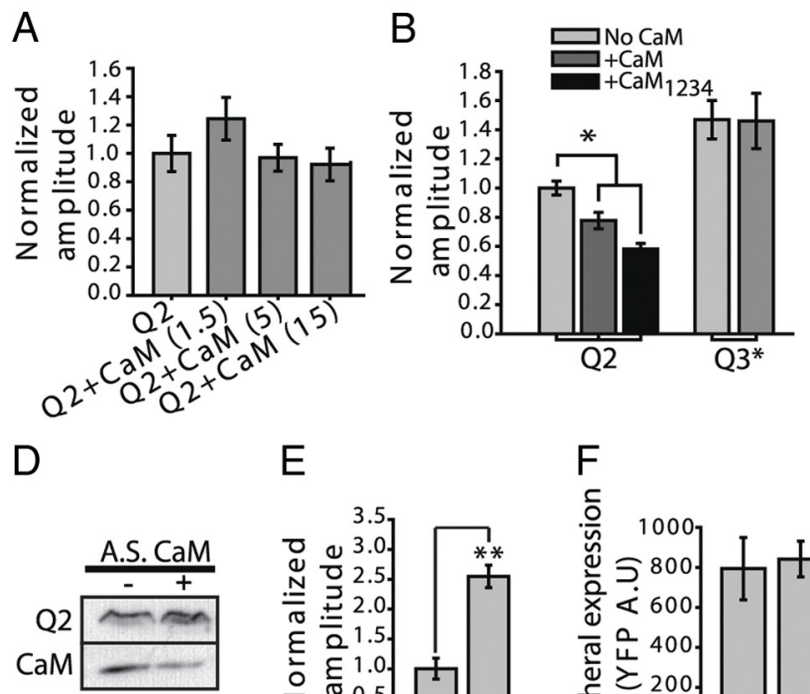

E

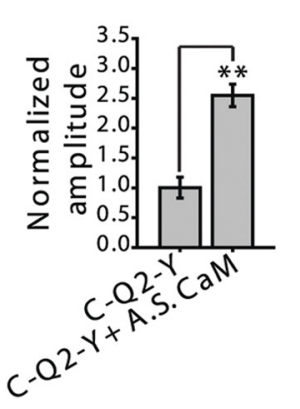

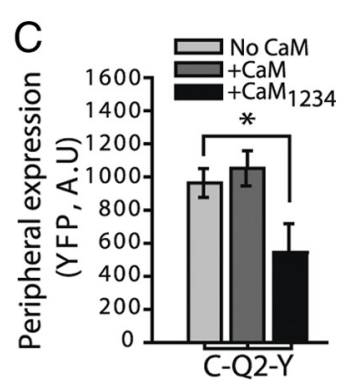

G

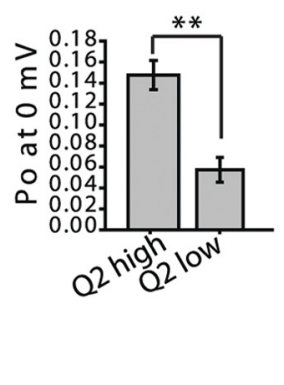

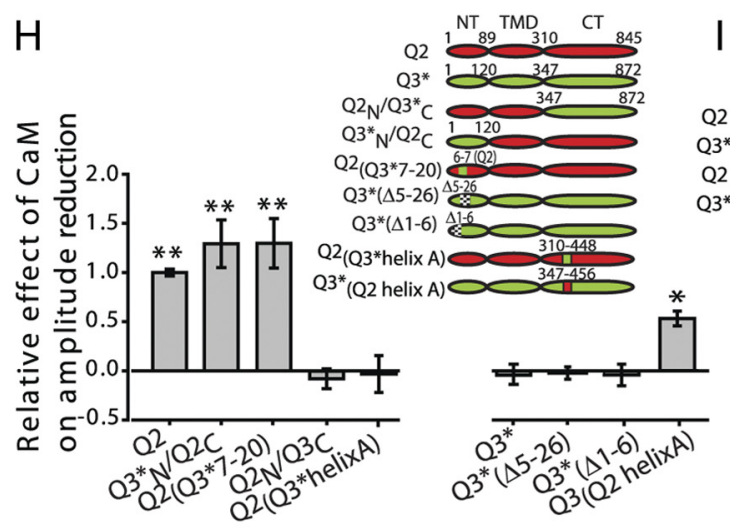

$J$

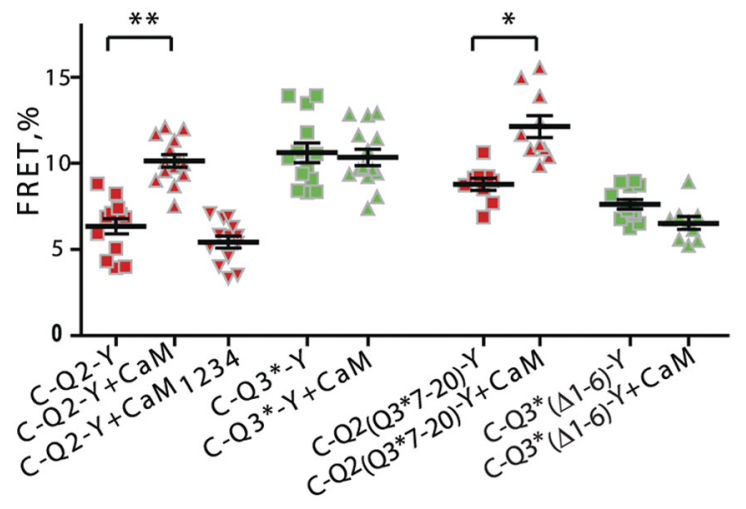

Q2 407 OKVSLKDRVFSS-PRGVAAKG 426 Q3* 422 QKLGLLDRVRISNPRGSNTKG 442 Q2 427 KGSPQAQTVRRSPSADQSLED 448 Q3*443 K-------LFTPLNVDAIEES 456
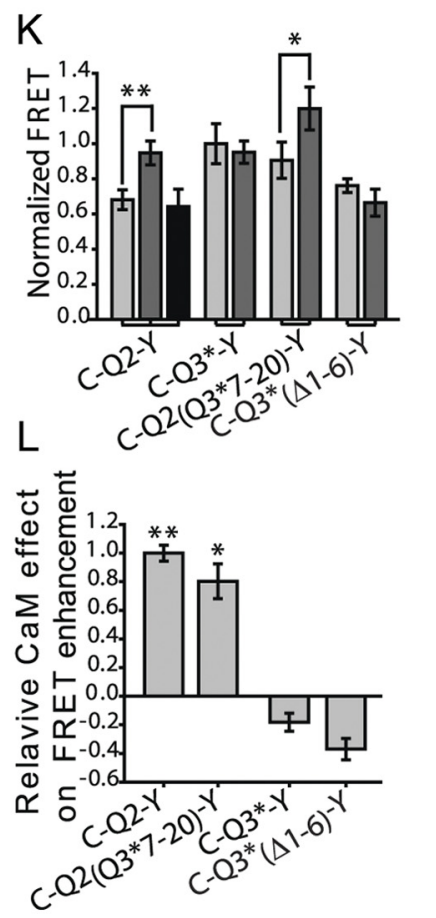

Figure 7. CaM decreases the current amplitudes of $Q 2$ but not of $Q 3^{*}$ and increases the FRET between the $\mathrm{N}$ and $\mathrm{C}$ termini of double fluorescent-labeled Q2 but not Q3*. The effect is dependent on the CT and not on the NT distal-end parts. $\boldsymbol{A}$, At a standard channel expression level, CaM does not reduce Q2 currents. 0ocytes were injected with $Q 2$ (standard concentrations, $7.5 \mathrm{ng}$ / oocyte), alone or together with increasing concentrations (1.5, 5, or $15 \mathrm{ng} / 00$ cyte) of CaM. Currents were evoked by a voltage step from a holding potential of -90 to $+5 \mathrm{mV}(n=17-21 ; N=2)$. $\boldsymbol{B}$, At a high channel expression level, $\mathrm{CaM}_{\text {and }} \mathrm{CaM}_{1234}$ reduce

Reciprocally, knockdown of endogenous $\mathrm{CaM}$ with antisense oligodeoxynucleotide for CaM (A.S. CaM) in oocytes expressing high channel levels (Fig. 7D) increased Q2 current amplitudes (Fig. $7 E)$, with no effect on channel peripheral expression (Fig. $7 F$ ). These results suggested that also endogenous CaM downregulates channel gating. To substantiate this notion, we tried to assess the impact of endogenous CaM on Po of Q2. We reasoned that changing the endogenous $\mathrm{CaM} / \mathrm{Q} 2$ ratio should result in different

the current amplitudes of $\mathrm{Q2}$ but not of $\mathrm{Q} 3^{*}$. Oocytes were injected with Q2 (20 ng/0ocyte) or Q3* (2 ng/00cyte) mRNAs, alone or together with equal amounts of $\mathrm{CaM}$ or $\mathrm{CaM}_{1234}$ mRNAs ( $n=9-35 ; N=1-3)$. C, $C_{2} M_{1234}$, but not CaM, reduces $C-02-Y$ peripheral expression. Peripheral levels were measured from confocal images of oocytes, taken under 514 $\mathrm{nm}$ laser excitations, in whole oocytes expressing $\mathrm{C}-\mathrm{Q} 2-\mathrm{Y}$, alone or together with $\mathrm{CaM}_{\text {or }} \mathrm{CaM}_{1234}(n=15)$. D, Endogenous CaM levels are knocked down in oocytes that were injected with antisense oligodeoxynucleotide targeted against CaM (A.S. CaM) $1 \mathrm{~d}$ before the channel injection and every $48 \mathrm{~h}$ until the assay. Western blot analysis of $\mathrm{C}-\mathrm{Q} 2-\mathrm{Y}$ (top panel) and CaM (bottom panel) from homogenates of oocytes injected with C-Q2-Y, alone or together with A.S. CaM, was performed using antibodies against $Q 2$ or CaM, respectively. C-Q2-Y expression levels were not reduced. $\boldsymbol{E}$, Antisense knockdown of endogenous CaM increases current amplitudes in oocytes expressing C-Q2-Y (45 ng/00cyte) injected with antisense oligodeoxynucleotide targeted against CaM (A.S. CaM) $1 \mathrm{~d}$ before the channel injection and every $48 \mathrm{~h}$ until the assay. Currents were evoked by a voltage step from a holding potential of -90 to $+5 \mathrm{mV}$. $\boldsymbol{F}$, Peripheral levels of $\mathrm{C}-02-\mathrm{Y}$ measured from confocal images taken under $514 \mathrm{~nm}$ laser excitations, in oocytes expressing $\mathrm{C}-\mathrm{Q} 2-\mathrm{Y}$, alone or together with A.S. CaM are similar ( $n=7$; same oocytes in $\boldsymbol{E}$ and $\boldsymbol{F}$ ). $\boldsymbol{G}$, Po values averaged from patches of oocytes injected with high (25 ng/ oocyte) or low (2.5 ng/00cyte) Q2 mRNA. Patches contained one to three channels $(n=8-13)$. $\boldsymbol{H}$, The relative effect of CaM on reduction of current amplitudes (quantified as the fraction of current reduced in the presence of $\mathrm{CaM}$, normalized to the fraction reduced by (aM in Q2) is independent of the NT distal-end parts, but is dependent on the $\mathrm{CT}$, in contrast to Syx ( $n=10-35 ; N=1-3)$. Left, The effect of CaM on 02 current amplitudes is resistant to changes in Q2NT but is lost upon exchange of Q2CT with $2^{*} \mathrm{CT}$; specifically, it is lost by swapping of the helix A domains. Right, $03^{*}$ does not gain CaM function by changes in its NT but only by exchange of its helix $A$ with that of Q2. Inset, Schematic representations of Q2, Q3*, and the chimeras. I, Alignment of Q2 (red) and Q3 (green) CT regions responsible for the isoform-specific effect of CaM. $\boldsymbol{J}-\boldsymbol{L}$, $\mathrm{CaM}$, but not $\mathrm{CaM}_{1324}$, increases the FRET in $\mathrm{C}-\mathrm{Q2}-\mathrm{Y}$, but not in $\mathrm{C}-\mathrm{Q} 3^{*}-\mathrm{Y}$. The $\mathrm{CaM}$-induced increase in FRET is independent of the NT distal-end parts, in contrast to Syx. Shown are a representative experiment showing FRETs in the absence and presence of $\mathrm{CaM}_{\text {or }} \mathrm{CaM}_{1234}(\boldsymbol{J} ; n=8-15)$; data were pooled from three experiments $(n=10-46)$ as in $J$, plotted as averaged FRETs that are normalized to that of $C-03^{*}-Y(K)$, and as relative effects of CaM on FRET enhancement (quantified as the fraction of FRET enhanced in the presence of $\mathrm{CaM}$, normalized to that of $Q 2 ; \boldsymbol{L}$ ). Note the correlation between the relative effects of CaM on FRET $(\boldsymbol{L})$ and on current amplitude $(\boldsymbol{H}) .{ }^{*} p<$ $0.05 ;{ }^{* *} p<0.01$. Error bars indicate SEM. 

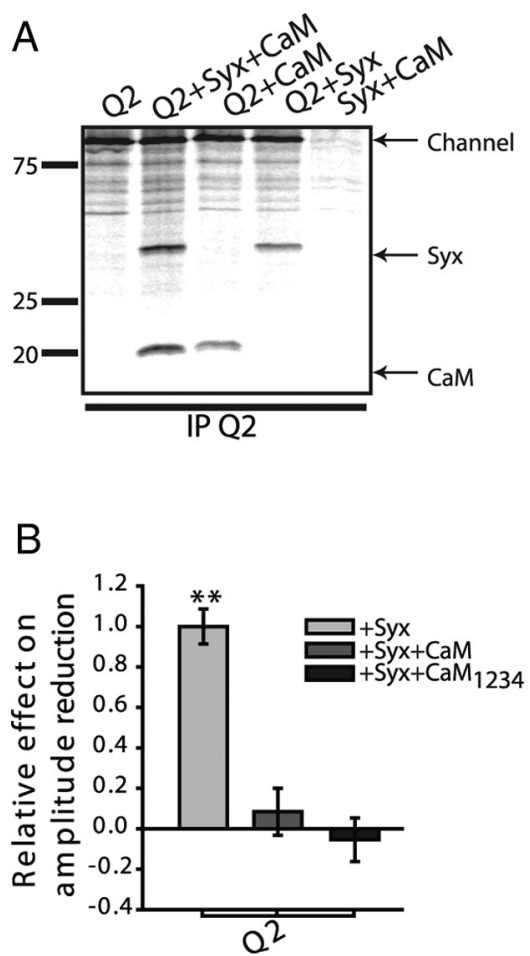

$\mathrm{D}$

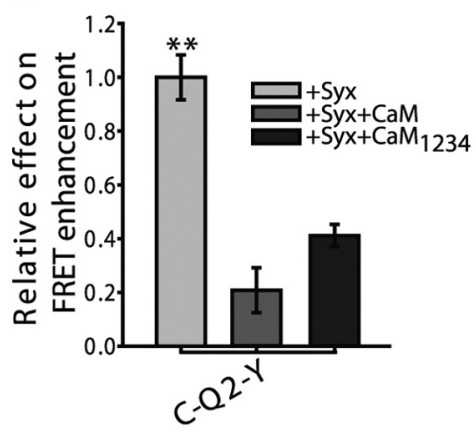

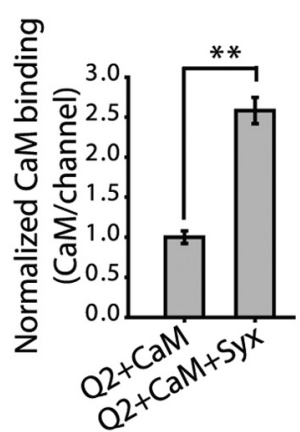
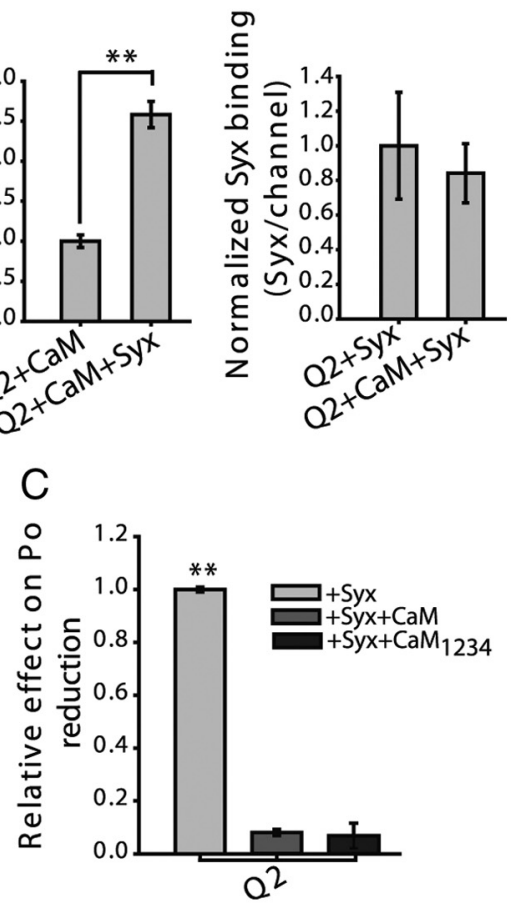

$E$

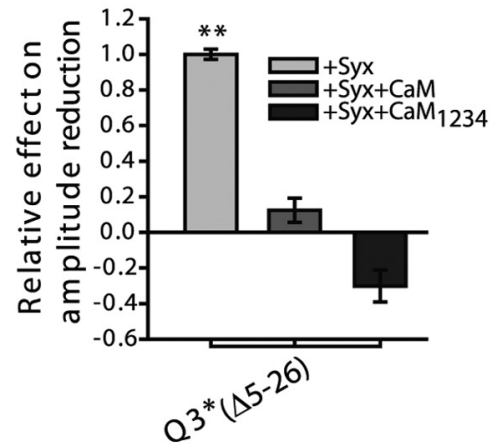

Figure 8. Coexpression of CaM with Syx results in elimination of the effect of Syx on current amplitude, $\mathrm{P} 0$, and N-Cinteraction. $A$, Syx enhances the binding of CaM to the channel. Left, Digitized Phosphorlmager scan of a coimmunoprecipitation analysis by SDS-PAGE of [ ${ }^{35}$ S]Met/Cys-labeled Q2, Syx, and CaM, using antibody against Q2, from homogenates of oocytes of a single frog injected with Q2, alone or in combination with Syx and/or CaM, mRNAs, as denoted above the lanes. The results shown are from one of three similar independent experiments. Middle and right, Summarized data of normalized CaM and Syx bindings, respectively, from three independent experiments. The bars depict band intensity ratios (quantified by ImageQuant) of CaM or Syx, coprecipitated with Q2, to precipitated Q2. $\boldsymbol{B}$, In the presence of $\mathrm{CaM}_{\text {or }} \mathrm{CaM}_{1234}$, the effect of Syx on Q2 current amplitude is blocked. Shown are the effects of Syx on current amplitudes in oocytes injected with Q2 (20 ng/0ocyte) together with Syx, alone or in combination with CaM or $\mathrm{CaM}_{1234}$ (as depicted below the bars), relative to that in oocytes injected with $\mathrm{Q2}$ and Syx. Currents were evoked by a

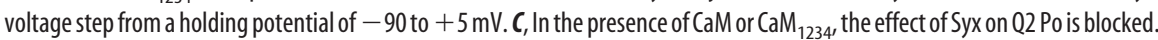
Shown are the effects of Syx on Po values derived from analysis of patches (containing 1-3 channels) of oocytes injected with Q2 together with Syx, alone or in combination with CaM (as depicted below the bars) relative to that in oocytes injected with Q2 and Syx $(n=8-10)$. $\boldsymbol{D}$, In the presence of CaM or $\mathrm{CaM}_{1234}$, the effect of Syx on FRET of $\mathrm{C}-\mathrm{Q2}-\mathrm{Y}$ is blocked $(n=10-24 ; N=1-2)$. Shown are the effects of Syx on FRETs in oocytes injected with Q2 together with Syx, alone or in combination with CaM or $\mathrm{CaM}_{1234}$ (as depicted below the bars), relative to that in oocytes injected with $\mathrm{Q2}$ and Syx. $\boldsymbol{E}$, In the presence of $\mathrm{CaM}_{\mathrm{M}}$ or $\mathrm{CaM}_{1234}$, the effect of Syx on $Q 3_{(\Delta 5-26)}^{*}\left(2 \mathrm{ng} / 00\right.$ cyte) current amplitudes is blocked. Experimental and presentation details are as in $\boldsymbol{B} .{ }^{* *} p<0.01$. Error bars indicate SEM.

Po levels, the higher the ratio, the lower Po, reflecting the tonic downregulation of channels by endogenous CaM. Thus, we performed analysis of single Q2 channels expressed from high and low mRNA concentrations, creating conditions of low and high endogenous CaM/Q2 ratios, respectively. Indeed, Po values of single channels expressed at low channel concentration were

significantly lower than those of channels expressed at the higher concentration (Fig. 7G), suggesting tonic downregulation of Q2 Po by endogenous CaM. Together, it was concluded that $\mathrm{CaM}$, endogenous or overexpressed, probably in a manner that requires its $\mathrm{Ca}^{2+}$. binding capacity, downregulates channel gating.

Next, we tested whether CaM-induced decrease of Q2 current amplitudes is linked to the NT distal-end parts using the NT chimeras. Strikingly, the CaM effect on Q2 was resistant to changes of the whole Q2 NT or its distal-end parts, but was abolished upon swapping Q2 CT with that of Q3 (Fig. 7H, left panel). Specifically, exchanging its helix A domain with that of Q3 caused loss of function to Q2 (Fig. $7 H$, left panel). Reciprocally, exchanging helix A domain of Q3 with that of Q2 caused gain of function to Q3* (Fig. $7 H$, right panel).

Next, we aimed to explore a possible role for CaM in the Q2 $\mathrm{N}-\mathrm{C}$ interaction by FRET analysis. Coexpressed CaM, but not $\mathrm{CaM}_{1234}$, significantly increased the FRET between the $\mathrm{N}$ and $\mathrm{C}$ termini of $\mathrm{C}-\mathrm{Q} 2-\mathrm{Y}$ but not of C-Q3*-Y (Fig. 7J,K). This substantiated the emerging notion (Fig. $7 B, C$ ) that $\mathrm{Ca}^{2+}$-binding capacity is needed for CaM-induced modulation. The CaMinduced increase of FRET was independent of the NT distal-end module, in contrast to Syx (Fig. 6A,B), as CaM increased the FRET of C-Q2 $\left.{ }_{(\mathrm{Q} 3} 7-20\right)^{-} \mathrm{Y}$ but failed to increase that of $\mathrm{C}-\mathrm{Q} 3^{*}(\Delta 1-6)-\mathrm{Y}$ (Fig. $7 J, K$ ). Hence, correlating with its gating effect, CaM effect on the $\mathrm{N}-\mathrm{C}$ interaction was determined by the CTs (Fig. 7, compare $H, L$ ). In summary, the structural determinants that confer $\mathrm{CaM}$ and Syx isoform selectivity with regard to both gating and $\mathrm{N}-\mathrm{C}$ modulations are different.

\section{Coexpression of CaM and Syx renders Q2 insensitive to their modulations}

Next, we tested a possible interplay between Syx and CaM, as both modulators share somewhat overlapping binding sites. We were encouraged by our co-IP analysis in oocytes coexpressing Q2 and various combinations of Syx and CaM, which showed that the binding of CaM to Q2 was increased in the presence of Syx (Fig. $8 A$ ), suggesting that Syx and CaM simultaneously occupy a single channel, rather than separate channels, and interact allosterically. Strikingly, although Syx alone exerted significant effects, coexpression of CaM with Syx blocked the effects of Syx on Q2 current amplitude (Fig. 8B), Po (Fig. 8C), and FRET (Fig. $8 D$ ). This phenomenon was also shared by $\mathrm{CaM}_{1234}$, as its coexpression with Syx resulted in no apparent 
effect on either current amplitude (Fig. $8 B$ ), Po (Fig. $8 C$ ), or FRET (Fig. $8 D$ ). These results determine that, in contrast to its action to modulate Q2 (Fig. 7), this aspect of CaM action that blocks the effect of Syx is not dependent on its $\mathrm{Ca}^{2+}$ binding capacity and is probably mediated by allosteric interaction. To substantiate the notion that the two actions of $\mathrm{CaM}$ are distinct and mediated via two different pathways, we used the channel Q3 ${ }_{(\Delta 5-26)}^{*}$, which is resistant to current amplitude reduction by CaM (Fig. $7 \mathrm{H}$, right), but is reduced by Syx (Fig. $3 B$, right). Notably, coexpression of CaM did block the reduction of $\mathrm{Q}^{*}(\Delta 5-26)$ currents by Syx (Fig. $8 E$ ).

\section{Discussion}

This study provides mechanistic insight into how Syx and CaM affect M-channel gating in an isoform-specific manner and puts forward a model for the regulation of gating. It reveals constitutive close association of channel cytoplasmic domains in Q2 and Q3; only in Q2 a specific rearrangement within this cytosolic structure, induced by Syx and CaM bindings, impacts on channel gating. Surprisingly, despite similarity in the Syx and CaM effects in Q2, these effects are prevented in Q3 by different $\mathrm{N}$ - and C-terminal structural determinants, suggesting that membrane-anchored Syx versus cytosolic CaM may induce different conformational changes to impart similar final arrangement of the termini resulting in reduced Po. Finally, we demonstrate a complex functional interplay between Syx and CaM, bearing potential physiological significance with regard to presynaptic release (see below).

\section{Closer proximity between the cytosolic} termini underlies $\mathbf{Q} 2$ gating downregulation

In search for determinants that impart the Q2-selective gating regulation by Syx, we identified NT domains (Figs. 3B, 4). Bearing in mind that Syx binds at the CTs (Regev et al., 2009), this suggested a possible cross talk between closely positioned $\mathrm{N}$ and $\mathrm{C}$ termini. Notably, $\mathrm{N}-\mathrm{C}$ close associations that are involved in the gating and activation of many channel types have been demonstrated (Varnum and Zagotta, 1997; Schulte et al., 1998; Tucker and Ashcroft, 1999; Qu et al., 2000; Lippiat et al., 2002; Tsuboi et al., 2004; Kobrinsky et al., 2006). Furthermore, using FRET analysis, our laboratory has recently demonstrated that a Kv2.1 regulatory protein modulates channel gating via effects on the association between the termini (Lvov et al., 2009). Here, pulldown assays (Fig. 5F) supported FRET analysis (Fig. 5D,E) demonstrating direct $\mathrm{N}-\mathrm{C}$ association in both Q2 and Q3. This finding, together with results of Q2/Q3 chimeras (Fig. 5E), led us to depict in a working model (Fig. $9 A-C$ ) constitutive $\mathrm{N}-\mathrm{C}$ interactions in both Q2 and Q3 channels. These interactions consist of a basal interaction (horizontal red lines; Fig. 9A), probably common to both channels,
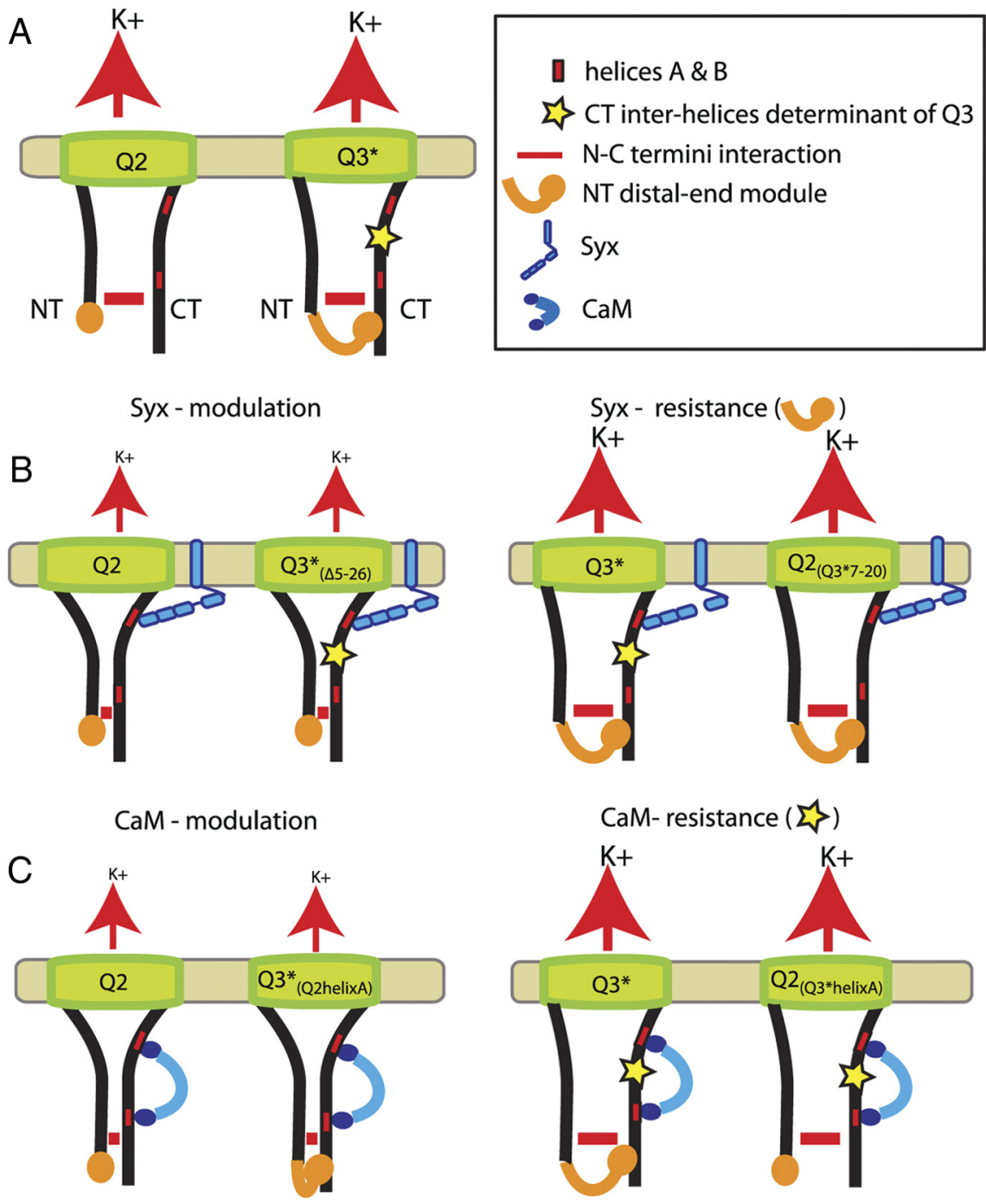

Figure 9. A-C, Model depicting $\mathrm{N}-\mathrm{C}$ interactions, constitutive and corresponding to gating modulations by Syx and CaM. A, Q2 and $\mathrm{Q3}$ (helices $\mathrm{C}$ and $\mathrm{D}$ in the CTs were omitted for clarity) carry constitutive $\mathrm{N}-\mathrm{C}$ interactions, inter or intra subunit. The interactions consist of a basal interaction, probably common to both channels (red horizontal lines) and an additional interaction proximity, accompanied by a decrease in Po (depicted by smaller vertical red arrows of $\mathrm{K}^{+}$fluxes). Resistance to Syx (B, right) or CaM (C, right) modulations is conferred by a Q3-specific NT (orange) or CT (yellow star) determinants, respectively.

and an additional interaction in Q3 formed by the NT distal-end module (orange; Fig. 9A). The $\mathrm{N}-\mathrm{C}$ interactions may be intermolecular or intramolecular.

Furthermore, Syx-induced downregulation of Q2 gating was accompanied by enhanced FRET (Fig. 6). We believe that the FRET change likely reports a proximity rather than orientation change of the fluorescent domains, since the FRET dependence on orientation is less a problem when fluorescent proteins are fused to the distal ends of channel termini and hence are relatively mobile (Zheng and Zagotta, 2004). Thus, in Figure 9B, left, which depicts our view of Syx modulation, increased $\mathrm{N}-\mathrm{C}$ proximity (decreased $\mathrm{N}-\mathrm{C}$ distance) accompanies the gating downregulation (smaller size of the red vertical arrow representing $\mathrm{K}^{+}$flux above channel). Notably, both Syx effects were manifested only in channels that do not have the NT distal-end module (Fig. 9B, left vs right), strongly arguing that this module prevents the effects of Syx in Q3.

Since CaM seemed to share similar characteristics with Syx in its interaction with the channels, binding at somewhat overlap- 
ping sites and selectively downregulating Q2 gating (Wen and Levitan, 2002; Yus-Najera et al., 2002; Gamper and Shapiro, 2003; Gamper et al., 2005; Bal et al., 2008; Regev et al., 2009), we expected to find similar functional modes for both regulators. Indeed, based on our results (Fig. 7) and sketched in Figure 9C, the gating downregulation by CaM is accompanied by increased $\mathrm{N}-\mathrm{C}$ proximity, similar to that by Syx. However, in contrast to Syx, the effects of CaM are indifferent to the NT distal-end module but are hampered by the Q3 CT structural determinant. These results are in accord with previous mapping of the effect of CaM on Q4 (using chimeras between Q3 and Q4) to the linker between helices A and B (Gamper et al., 2005). Since in our constructs, used for the mapping of the effect of CaM, the exchanged helix A domains also included a segment of the linkers, we mapped (Fig. $7 I$ ) the effect of CaM, by aligning with the region identified previously (Gamper et al., 2005), to amino acids Q407-D448 and Q422-S456 in the linker between helices A and B of Q2 and Q3, respectively (the Q3 interhelices determinant that hampers the CaM effects is represented as yellow star in Fig. 9).

In this context, it is intriguing that the effect of $\mathrm{Ca}^{2+}-\mathrm{CaM}$ is mapped to a site at the linker between the binding sites of CaM, helix A and B. It is possible that the conformational change of the $\mathrm{CaM} /$ channel complex upon $\mathrm{Ca}^{2+}$ binding involves the linker, which, in turn, affects the interaction of the $\mathrm{C}$ terminus with the $\mathrm{N}$ terminus. Notably, there is only 10 aa proximity between the CaM modulatory site and that of $\mathrm{PIP}_{2}$ (Hernandez et al., 2008); both modulators downregulate channel gating (Gamper et al., 2005; Li et al., 2005; Hernandez et al., 2008), possibly suggesting that also the effect of PIP2 is mediated via N-C interaction.

The strong correlation between enhanced FRET and gating downregulation by both Syx and CaM (Figs. 6C,D, 7 H, L, respectively) strongly argues for a causal relationship between closer $\mathrm{N}-\mathrm{C}$ proximity and downregulated gating.

\section{Interplay between Syx and CaM in Q2 gating modulation}

Syx and CaM seem to simultaneously associate with a single channel, rather than separate channels (Fig. $8 A$ ). This, together with the similarities and dissimilarities in their modes of action, imply a complex functional relationship between Syx and CaM. We suggest two modes of interplay between the Syx and CaM, subject to the level of expression of $\mathrm{CaM}$ and its ability to associate with $\mathrm{Ca}^{2+}$. In one mode, downregulations of channel gating by CaM and Syx are additive and mediated via two distinct binding sites. This relationship was manifested in our system at standard Q2 expression level when channels saturated with endogenous $\mathrm{CaM}$ did not respond to overexpressed CaM (Fig. 7A) but were still downregulated by overexpressed Syx (Figs. 1, 2B-D). This mode requires the $\mathrm{Ca}^{2+}$-binding capacity of CaM (Fig. $7 B, H$ ). In the second mode, overexpressed CaM acts to block the effects of Syx, independent of its $\mathrm{Ca}^{2+}$ binding capacity (Fig. $8 B-D$ ). A plausible possibility is that two distinct CaM binding sites mediate the two modes, a "high" affinity site that is saturated at resting conditions by the endogenous CaM and mediates Q2 gating downregulation, and a "low" affinity site that mediates the blockade of the gating downregulation by Syx. In the second mode, the CaM blockade of Syx action may involve allosteric hindrance caused by CaM overoccupancy, possibly due to the well known target promiscuity of CaM. The distinction between the two modes is underscored by the mutant channel that is resistant to CaM modulation, but its modulation by Syx is still blocked by CaM (Fig. 8E).

\section{Physiological significance of the Syx and CaM modulations}

Q2 but not Q3 subunits have been suggested to be involved in the direct regulation of neurotransmitter release by M-channels at the nerve terminal (Martire et al., 2004; Peretz et al., 2007). In contrast, Q2/Q3 heteromeric channels are predominant at the neuron initial segment and regulate neuronal firing frequency (Devaux et al., 2004; Chung et al., 2006). Hence, the selective downregulation of $\mathrm{Q} 2$, and not $\mathrm{Q} 3$, gating by regulatory proteins may be targeted toward homomeric Q2 channels so as to finetune presynaptic transmitter release without interfering with the function of heteromeric channels in neuronal firing frequency adaptation (Regev et al., 2009).

Both Syx and CaM can act presynaptically and may interact with Q2 subunits at nerve terminals. Syx has been shown to colocalize with and to bind Q2-containing channels at hippocampal presynaptic boutons and in synaptosomal membranes (Regev et al., 2009). CaM may regulate neurotransmitter release through modulation of presynaptic M-current, either by control of axonal action potential firing or via direct effect on presynaptic M-channels (Martire et al., 2004, 2007; Peretz et al., 2007; Hernandez et al., 2008). One such direct mechanism could be mediated by $\mathrm{Ca}^{2+}$ activation of $\mathrm{CaM}$ bound to $\mathrm{M}$-channels through presynaptic bradykinin receptor activation (Trendelenburg et al., 2000).

This study suggests an intricate interplay between the two proteins, CaM and Syx, which may have significance in the regulation of neurotransmitter release under normal and pathological conditions. Under normal physiological activity, the additive nature of the CaM and Syx modulations may serve as a mechanism to enforce robust M-channel activity suppression, enhancing neurotransmitter release. However, under pathophysiological conditions, such as ischemia (Palfi et al., 2001), posttranscriptional regulation of CaM may occur and lead to upregulation of CaM levels (Vizi et al., 2000; Palfi et al., 2002; Kortvely and Gulya, 2004), shown here to block the suppression of M-channels. Notably, suppression of M-channels by bradykinin $\mathrm{B}_{2}$ receptor stimulation was shown to be blocked by heterologous expression of WT CaM in superior cervical ganglions (Zaika et al., 2007). Hence, the CaM blocking of M-channel suppression is a plausible neuronal mechanism to alleviate potassium current and reduce local excitability and transmitter release under pathological conditions that provide the tissue too little oxygen for its metabolic needs.

\section{References}

Bal M, Zaika O, Martin P, Shapiro MS (2008) Calmodulin binding to M-type $\mathrm{K}^{+}$channels assayed by TIRF/FRET in living cells. J Physiol 586:2307-2320.

Biervert C, Schroeder BC, Kubisch C, Berkovic SF, Propping P, Jentsch TJ, Steinlein OK (1998) A potassium channel mutation in neonatal human epilepsy. Science 279:403-406.

Bofill-Cardona E, Kudlacek O, Yang Q, Ahorn H, Freissmuth M, Nanoff C (2000) Binding of calmodulin to the D2-dopamine receptor reduces receptor signaling by arresting the $\mathrm{G}$ protein activation switch. J Biol Chem 275:32672-32680.

Bright JN, Sansom MSP (2003) The flexing/twirling helix: exploring the flexibility about molecular hinges formed by praline and glycine motifs in transmembrane helices. J Phys Chem B 107:627-636.

Brown DA, Adams PR (1980) Muscarinic suppression of a novel voltagesensitive $\mathrm{K}^{+}$current in a vertebrate neurone. Nature 283:673-676.

Cartaud A, Ozon R, Walsh MP, Haiech J, Demaille JG (1980) Xenopus laevis oocyte calmodulin in the process of meiotic maturation. J Biol Chem 255:9404-9408.

Chien YH, Dawid IB (1984) Isolation and characterization of calmodulin genes from Xenopus laevis. Mol Cell Biol 4:507-513.

Chung HJ, Jan YN, Jan LY (2006) Polarized axonal surface expression of neuronal KCNQ channels is mediated by multiple signals in the KCNQ2 
and KCNQ3 C-terminal domains. Proc Natl Acad Sci USA 103:8870-8875.

Cooper EC, Jan LY (2003) M-channels: neurological diseases, neuromodulation, and drug development. Arch Neurol 60:496-500.

Cooper EC, Harrington E, Jan YN, Jan LY (2001) M channel KCNQ2 subunits are localized to key sites for control of neuronal network oscillations and synchronization in mouse brain. J Neurosci 21:9529-9540.

Cruzblanca H, Koh DS, Hille B (1998) Bradykinin inhibits M current via phospholipase $\mathrm{C}$ and $\mathrm{Ca}^{2+}$ release from IP3-sensitive $\mathrm{Ca}^{2+}$ stores in rat sympathetic neurons. Proc Natl Acad Sci U S A 95:7151-7156.

Dascal N (1993) Expression of exogenous ion channels and neurotransmitter receptors in RNA-injected Xenopus oocytes. Methods Mol Biol 13:205-225.

Delmas P, Brown DA (2005) Pathways modulating neural KCNQ/M (Kv7) potassium channels. Nat Rev Neurosci 6:850-862.

Devaux JJ, Kleopa KA, Cooper EC, Scherer SS (2004) KCNQ2 is a nodal $\mathrm{K}^{+}$ channel. J Neurosci 24:1236-1244.

Dolly JO, Parcej DN (1996) Molecular properties of voltage-gated $\mathrm{K}^{+}$channels. J Bioenerg Biomembr 28:231-253.

Etxeberria A, Santana-Castro I, Regalado MP, Aivar P, Villarroel A (2004) Three mechanisms underlie KCNQ2/3 heteromeric potassium M-channel potentiation. J Neurosci 24:9146-9152.

Etxeberria A, Aivar P, Rodriguez-Alfaro JA, Alaimo A, Villacé P, GómezPosada JC, Areso P, Villarroel A (2008) Calmodulin regulates the trafficking of KCNQ2 potassium channels. FASEB J 22:1135-1143.

Fili O, Michaelevski I, Bledi Y, Chikvashvili D, Singer-Lahat D, Boshwitz H, Linial M, Lotan I (2001) Direct interaction of a brain voltage-gated $\mathrm{K}^{+}$ channel with syntaxin 1A: functional impact on channel gating. J Neurosci 21:1964-1974.

Ford CP, Stemkowski PL, Light PE, Smith PA (2003) Experiments to test the role of phosphatidylinositol 4,5-bisphosphate in neurotransmitterinduced M-channel closure in bullfrog sympathetic neurons. J Neurosci 23:4931-4941.

Gamper N, Shapiro MS (2003) Calmodulin mediates $\mathrm{Ca}^{2+}$-dependent modulation of M-type $\mathrm{K}^{+}$channels. J Gen Physiol 122:17-31.

Gamper N, Li Y, Shapiro MS (2005) Structural requirements for differential sensitivity of $\mathrm{KCNQ} \mathrm{K}^{+}$channels to modulation by $\mathrm{Ca}^{2+} /$ calmodulin. Mol Biol Cell 16:3538-3551.

Gao Y, Liu SS, Qiu S, Cheng W, Zheng J, Luo JH (2007) Fluorescence resonance energy transfer analysis of subunit assembly of the ASIC channel. Biochem Biophys Res Commun 359:143-150.

Geiser JR, van Tuinen D, Brockerhoff SE, Neff MM, Davis TN (1991) Can calmodulin function without binding calcium? Cell 65:949-959.

Griesbeck O, Baird GS, Campbell RE, Zacharias DA, Tsien RY (2001) Reducing the environmental sensitivity of yellow fluorescent protein. Mechanism and applications. J Biol Chem 276:29188-29194.

Hernandez CC, Zaika O, Tolstykh GP, Shapiro MS (2008) Regulation of neural KCNQ channels: signalling pathways, structural motifs and functional implications. J Physiol 586:1811-1821.

Hille B (1992) Ion channels of excitable membranes. Sunderland, MA: Sinauer Associates.

Jentsch TJ (2000) Neuronal KCNQ potassium channels: physiology and role in disease. Nat Rev Neurosci 1:21-30.

Jing J, Chikvashvili D, Singer-Lahat D, Thornhill WB, Reuveny E, Lotan I (1999) Fast inactivation of a brain $\mathrm{K}^{+}$channel composed of Kv1.1 and Kvbeta 1.1 subunits modulated by $\mathrm{G}$ protein beta gamma subunits. EMBO J 18:1245-1256.

Kobrinsky E, Stevens L, Kazmi Y, Wray D, Soldatov NM (2006) Molecular rearrangements of the Kv2.1 potassium channel termini associated with voltage gating. J Biol Chem 281:19233-19240.

Kortvely E, Gulya K (2004) Calmodulin, and various ways to regulate its activity. Life Sci 74:1065-1070.

Leung YM, Kang Y, Gao X, Xia F, Xie H, Sheu L, Tsuk S, Lotan I, Tsushima RG, Gaisano HY (2003) Syntaxin 1A binds to the cytoplasmic C terminus of Kv2.1 to regulate channel gating and trafficking. J Biol Chem 278:17532-17538.

Leung YM, Kwan EP, Ng B, Kang Y, Gaisano HY (2007) SNAREing voltagegated $\mathrm{K}^{+}$and ATP-sensitive $\mathrm{K}^{+}$channels: tuning beta-cell excitability with syntaxin-1A and other exocytotic proteins. Endocr Rev 28:653-663.

Levin G, Keren T, Peretz T, Chikvashvili D, Thornhill WB, Lotan I (1995) Regulation of RCK1 currents with a cAMP analog via enhanced protein synthesis and direct channel phosphorylation. J Biol Chem 270:14611-14618.

Li SC, Deber CM (1992) Glycine and beta-branched residues support and modulate peptide helicity in membrane environments. FEBS Lett 311:217-220.

Li Y, Langlais P, Gamper N, Liu F, Shapiro MS (2004) Dual phosphorylations underlie modulation of unitary $\mathrm{KCNQ} \mathrm{K}^{+}$channels by Src tyrosine kinase. J Biol Chem 279:45399-45407.

Li Y, Gamper N, Hilgemann DW, Shapiro MS (2005) Regulation of Kv7 (KCNQ) $\mathrm{K}^{+}$channel open probability by phosphatidylinositol 4,5bisphosphate. J Neurosci 25:9825-9835.

Lippiat JD, Albinson SL, Ashcroft FM (2002) Interaction of the cytosolic domains of the Kir6.2 subunit of the $\mathrm{K}_{\mathrm{ATP}}$ channel is modulated by sulfonylureas. Diabetes 51 [Suppl 3]:S377-S380.

Lvov A, Greitzer D, Berlin S, Chikvashvili D, Tsuk S, Lotan I, Michaelevski I (2009) Rearrangements in the relative orientation of cytoplasmic domains induced by a membrane-anchored protein mediate modulations in Kv channel gating. J Biol Chem 284:28276-28291.

Marrion NV (1997) Control of M-current. Annu Rev Physiol 59:483-504.

Martire M, Castaldo P, D’Amico M, Preziosi P, Annunziato L, Taglialatela M (2004) M channels containing KCNQ2 subunits modulate norepinephrine, aspartate, and GABA release from hippocampal nerve terminals. J Neurosci 24:592-597.

Martire M, D’Amico M, Panza E, Miceli F, Viggiano D, Lavergata F, Iannotti FA, Barrese V, Preziosi P, Annunziato L, Taglialatela M (2007) Involvement of KCNQ2 subunits in $\left[{ }^{3} \mathrm{H}\right]$ dopamine release triggered by depolarization and pre-synaptic muscarinic receptor activation from rat striatal synaptosomes. J Neurochem 102:179-193.

Michaelevski I, Chikvashvili D, Tsuk S, Singer-Lahat D, Kang Y, Linial M, Gaisano HY, Fili O, Lotan I (2003) Direct interaction of target SNAREs with the Kv2.1 channel. Modal regulation of channel activation and inactivation gating. J Biol Chem 278:34320-34330.

Michaelevski I, Korngreen A, Lotan I (2007) Interaction of syntaxin with a single Kv1.1 channel: a possible mechanism for modulating neuronal excitability. Pflugers Arch 454:477-494.

Millar ID, Bruce JIE, Brown PD (2007) Ion channel diversity, channel expression and function in the choroid plexuses. Cerebrospinal Fluid Res $4: 8$.

Miller C (2000) An overview of the potassium channel family. Genome Biol 1:REVIEWS0004.

Palfi A, Simonka JA, Pataricza M, Tekulics P, Lepran I, Papp G, Gulya K (2001) Postischemic calmodulin gene expression in the rat hippocampus. Life Sci 68:2373-2381.

Palfi A, Kortvely E, Fekete E, Kovacs B, Varszegi S, Gulya K (2002) Differential calmodulin gene expression in the rodent brain. Life Sci 70:2829-2855.

Peretz A, Sheinin A, Yue C, Degani-Katzav N, Gibor G, Nachman R, Gopin A, Tam E, Shabat D, Yaari Y, Attali B (2007) Pre- and postsynaptic activation of M-channels by a novel opener dampens neuronal firing and transmitter release. J Neurophysiol 97:283-295.

Qu Z, Yang Z, Cui N, Zhu G, Liu C, Xu H, Chanchevalap S, Shen W, Wu J, Li $\mathrm{Y}$, Jiang C (2000) Gating of inward rectifier $\mathrm{K}^{+}$channels by protonmediated interactions of $\mathrm{N}$ - and C-terminal domains. J Biol Chem 275:31573-31580.

Regev N, Degani-Katzav N, Korngreen A, Etzioni A, Siloni S, Alaimo A, Chikvashvili D, Villarroel A, Attali B, Lotan I (2009) Selective interaction of syntaxin 1A with KCNQ2: possible implications for specific modulation of presynaptic activity. PLoS One 4:e6586.

Robbins J (2001) KCNQ potassium channels: physiology, pathophysiology, and pharmacology. Pharmacol Ther 90:1-19.

Rubinstein M, Peleg S, Berlin S, Brass D, Keren-Raifman T, Dessauer CW, Ivanina T, Dascal N (2009) Divergent regulation of GIRK1 and GIRK2 subunits of the neuronal $\mathrm{G}$ protein gated $\mathrm{K}^{+}$channel by GalphaiGDP and Gbetagamma. J Physiol 587:3473-3491.

Schulte U, Hahn H, Wiesinger H, Ruppersberg JP, Fakler B (1998) pHdependent gating of ROMK (Kir1.1) channels involves conformational changes in both $\mathrm{N}$ and $\mathrm{C}$ termini. J Biol Chem 273:34575-34579.

Schwake M, Pusch M, Kharkovets T, Jentsch TJ (2000) Surface expression and single channel properties of KCNQ2/KCNQ3, M-type $\mathrm{K}^{+}$channels involved in epilepsy. J Biol Chem 275:13343-13348.

Selyanko AA, Hadley JK, Brown DA (2001) Properties of single M-type 
KCNQ2/KCNQ3 potassium channels expressed in mammalian cells. J Physiol 534:15-24.

Shaner NC, Steinbach PA, Tsien RY (2005) A guide to choosing fluorescent proteins. Nat Methods 2:905-909.

Singer-Lahat D, Dascal N, Lotan I (1999) Modal behavior of the Kv1.1 channel conferred by the Kvbetal.1 subunit and its regulation by dephosphorylation of Kv1.1. Pflugers Arch 439:18-26.

Singh NA, Charlier C, Stauffer D, DuPont BR, Leach RJ, Melis R, Ronen GM, Bjerre I, Quattlebaum T, Murphy JV, McHarg ML, Gagnon D, Rosales TO, Peiffer A, Anderson VE, Leppert M (1998) A novel potassium channel gene, KCNQ2, is mutated in an inherited epilepsy of newborns. Nat Genet 18:25-29.

Suh BC, Hille B (2002) Recovery from muscarinic modulation of M current channels requires phosphatidylinositol 4,5-bisphosphate synthesis. Neuron 35:507-520.

Suh BC, Horowitz LF, Hirdes W, Mackie K, Hille B (2004) Regulation of $\mathrm{KCNQ} 2 / \mathrm{KCNQ} 3$ current by $\mathrm{G}$ protein cycling: the kinetics of receptormediated signaling by Gq. J Gen Physiol 123:663-683.

Takanishi CL, Bykova EA, Cheng W, Zheng J (2006) GFP-based FRET analysis in live cells. Brain Res 1091:132-139.

Trendelenburg AU, Cox SL, Schelb V, Klebroff W, Khairallah L, Starke K (2000) Modulation of ${ }^{3} \mathrm{H}$-noradrenaline release by presynaptic opioid, cannabinoid and bradykinin receptors and beta-adrenoceptors in mouse tissues. Br J Pharmacol 130:321-330.

Tsuboi T, Lippiat JD, Ashcroft FM, Rutter GA (2004) ATP-dependent interaction of the cytosolic domains of the inwardly rectifying $\mathrm{K}^{+}$channel Kir6.2 revealed by fluorescence resonance energy transfer. Proc Natl Acad Sci U S A 101:76-81.

Tsuk S, Michaelevski I, Bentley GN, Joho RH, Chikvashvili D, Lotan I (2005) Kv2.1 channel activation and inactivation is influenced by physical interactions of both syntaxin $1 \mathrm{~A}$ and the syntaxin $1 \mathrm{~A} /$ soluble $N$-ethylmaleimide-sensitive factor-25 (t-SNARE) complex with the $\mathrm{C}$ terminus of the channel. Mol Pharmacol 67:480-488.

Tucker SJ, Ashcroft FM (1999) Mapping of the physical interaction between the intracellular domains of an inwardly rectifying potassium channel, Kir6.2. J Biol Chem 274:33393-33397.

Varnum MD, Zagotta WN (1997) Interdomain interactions underlying activation of cyclic nucleotide-gated channels. Science 278:110-113.
Vizi S, Palfi A, Gulya K (2000) Multiple calmodulin genes exhibit systematically differential responses to chronic ethanol treatment and withdrawal in several regions of the rat brain. Brain Res Mol Brain Res 83:63-71.

Wang HS, Pan Z, Shi W, Brown BS, Wymore RS, Cohen IS, Dixon JE, McKinnon D (1998) KCNQ2 and KCNQ3 potassium channel subunits: molecular correlates of the M-channel. Science 282:1890-1893.

Weiner MP (1993) Directional cloning of blunt-ended PCR products. Biotechniques 15:502-505.

Wen H, Levitan IB (2002) Calmodulin is an auxiliary subunit of KCNQ2/3 potassium channels. J Neurosci 22:7991-8001.

Wiener R, Haitin Y, Shamgar L, Fernández-Alonso MC, Martos A, ChomskyHecht O, Rivas G, Attali B, Hirsch JA (2008) The KCNQ1 (Kv7.1) $\mathrm{COOH}$ terminus, a multitiered scaffold for subunit assembly and protein interaction. J Biol Chem 283:5815-5830.

Winks JS, Hughes S, Filippov AK, Tatulian L, Abogadie FC, Brown DA, Marsh SJ (2005) Relationship between membrane phosphatidylinositol-4,5bisphosphate and receptor-mediated inhibition of native neuronal $\mathrm{M}$ channels. J Neurosci 25:3400-3413.

Wurch T, Lestienne F, Pauwels PJ (1998) A modified overlap extension PCR method to create chimeric genes in the absence of restriction enzymes. Biotechnol Tech 12:653-657.

Yamakawa T, Saith S, Li Y, Gao X, Gaisano HY, Tsushima RG (2007) Interaction of syntaxin $1 \mathrm{~A}$ with the $\mathrm{N}$-terminus of Kv4.2 modulates channel surface expression and gating. Biochemistry 46:10942-10949.

Yus-Najera E, Santana-Castro I, Villarroel A (2002) The identification and characterization of a noncontinuous calmodulin-binding site in noninactivating voltage-dependent KCNQ potassium channels. J Biol Chem 277:28545-28553.

Zaika O, Tolstykh GP, Jaffe DB, Shapiro MS (2007) Inositol triphosphatemediated $\mathrm{Ca}^{2+}$ signals direct purinergic $\mathrm{P} 2 \mathrm{Y}$ receptor regulation of neuronal ion channels. J Neurosci 27:8914-8926.

Zhang H, Craciun LC, Mirshahi T, Rohács T, Lopes CM, Jin T, Logothetis DE (2003) PIP $_{2}$ activates KCNQ channels, and its hydrolysis underlies receptor-mediated inhibition of M currents. Neuron 37:963-975.

Zheng J, Zagotta WN (2004) Stoichiometry and assembly of olfactory cyclic nucleotide-gated channels. Neuron 42:411-421. 